\title{
Kirchhoff equations with strong damping
}

\author{
Marina Ghisi \\ Università degli Studi di Pisa \\ Dipartimento di Matematica \\ PISA (Italy) \\ e-mail: ghisi@dm.unipi.it \\ Massimo Gobbino \\ Università degli Studi di Pisa \\ Dipartimento di Matematica \\ PISA (Italy) \\ e-mail: massimo.gobbino@unipi.it
}




\begin{abstract}
We consider Kirchhoff equations with strong damping, namely with a friction term which depends on a power of the "elastic" operator. We address local and global existence of solutions in two different regimes depending on the exponent in the friction term.

When the exponent is greater than $1 / 2$, the dissipation prevails, and we obtain global existence in the energy space assuming only degenerate hyperbolicity and continuity of the nonlinear term. When the exponent is less than $1 / 2$, we assume strict hyperbolicity and we consider a phase space depending on the continuity modulus of the nonlinear term and on the exponent in the damping. In this phase space we prove local existence, and global existence if initial data are small enough.

The regularity we assume both on initial data and on the nonlinear term is weaker than in the classical results for Kirchhoff equations with standard damping.

Proofs exploit some recent sharp results for the linearized equation and suitably defined interpolation spaces.
\end{abstract}

Mathematics Subject Classification 2010 (MSC2010): 35L70, 35L80, 35L90.

Key words: quasilinear hyperbolic equation, degenerate hyperbolic equation, Kirchhoff equation, global existence, strong damping, fractional damping, interpolation spaces. 


\section{Introduction}

Let $H$ be a separable real Hilbert space. For every $x$ and $y$ in $H$, let $|x|$ denote the norm of $x$, and let $\langle x, y\rangle$ denote the scalar product of $x$ and $y$. Let $A$ be a self-adjoint linear operator on $H$ with dense domain $D(A)$. We assume that $A$ is nonnegative, namely $\langle A x, x\rangle \geq 0$ for every $x \in D(A)$, so that for every $\alpha \geq 0$ the power $A^{\alpha} x$ is defined provided that $x$ lies in a suitable domain $D\left(A^{\alpha}\right)$.

We consider the second order evolution equation

$$
u^{\prime \prime}(t)+2 \delta A^{\sigma} u^{\prime}(t)+m\left(\left|A^{1 / 2} u(t)\right|^{2}\right) A u(t)=0
$$

with initial data

$$
u(0)=u_{0}, \quad u^{\prime}(0)=u_{1},
$$

where $\delta>0$ and $\sigma \geq 0$ are real numbers, and $m:[0,+\infty) \rightarrow[0,+\infty)$ is a given function. Equations of this type have long been studied in the literature after Kirchhoff's monograph [26]. Let us give a brief outline of the previous results which are closely related to ours.

Non-dissipative Kirchhoff equations When $\delta=0$, equation (1.1) reduces to

$$
u^{\prime \prime}(t)+m\left(\left|A^{1 / 2} u(t)\right|^{2}\right) A u(t)=0 .
$$

This is the abstract setting of the hyperbolic partial differential equation

$$
u_{t t}(x, t)-m\left(\int_{\Omega}|\nabla u(x, t)|^{2} d x\right) \Delta u(x, t)=0,
$$

with suitable boundary conditions in an open set $\Omega \subseteq \mathbb{R}^{n}$. When $n=1$ or $n=2$, and $\Omega$ is bounded, this equation is a possible model for transversal small-amplitude vibrations of elastic strings or membranes. The nonlocal model was derived by Kirchhoff in [26] after some mathematical simplifications to the full system of (local) equations of nonlinear elasticity.

From the mathematical point of view, local existence for (1.3)-(1.2) is known provided that the nonlinearity $m(x)$ is Lipschitz continuous and satisfies the strict hyperbolicity condition

$$
m(x) \geq \mu_{1}>0 \quad \forall x \geq 0,
$$

and initial data $\left(u_{0}, u_{1}\right)$ are in the space $D\left(A^{3 / 4}\right) \times D\left(A^{1 / 4}\right)$. This result was substantially established by Bernstein in the pioneering paper [3], and then refined by many authors (see [1] for a modern version). Local existence results extend easily to the so-called mildly degenerate case, namely when the nonlinear term satisfies just the degenerate hyperbolicity condition

$$
m(x) \geq 0 \quad \forall x \geq 0
$$

but $m\left(\left|A^{1 / 2} u_{0}\right|^{2}\right)>0$. 
Global existence has been proved in a multitude of heterogeneous special situations, such as analytic data $([3,2,9,10])$, quasi-analytic data $([30,18])$, special nonlinearities ([35]), dispersive equations and small data $([21,11,40,29])$, spectral gap data or spectral gap operators $([27,23,15,18])$.

Global existence in Sobolev spaces is no doubt the main open problem in the theory of Kirchhoff equations. Local existence in Sobolev spaces is an open problem as well in at least three situations: when the nonlinear term is not Lipschitz continuous, when $m\left(\left|A^{1 / 2} u_{0}\right|^{2}\right)=0$ (the so-called really degenerate case, see [39]), and when initial data are just in the energy space $D\left(A^{1 / 2}\right) \times H$ or in any space weaker that $D\left(A^{3 / 4}\right) \times D\left(A^{1 / 4}\right)$. The interested reader is referred to the survey [17] for more details.

From the technical point of view, a key role is played by the linearization of (1.3), namely equation

$$
u^{\prime \prime}(t)+c(t) A u(t)=0,
$$

where $c(t)$ is a time-dependent coefficient. It is well-known that (1.6) admits global solutions for all initial data in the energy space $D\left(A^{1 / 2}\right) \times H$, or more generally in $D\left(A^{\alpha+1 / 2}\right) \times D\left(A^{\alpha}\right)$, provided that $c(t)$ is Lipschitz continuous and satisfies the strict hyperbolicity condition

$$
0<\mu_{1} \leq c(t) \leq \mu_{2}
$$

In the case of Kirchhoff equations the coefficient $c(t)$ is $m\left(\left|A^{1 / 2} u(t)\right|^{2}\right)$, hence its Lipschitz continuity is related to the boundedness of

$$
c^{\prime}(t)=m^{\prime}\left(\left|A^{1 / 2} u(t)\right|^{2}\right) \cdot 2\left\langle A u(t), u^{\prime}(t)\right\rangle=2 m^{\prime}\left(\left|A^{1 / 2} u(t)\right|^{2}\right) \cdot\left\langle A^{3 / 4} u(t), A^{1 / 4} u^{\prime}(t)\right\rangle .
$$

This is the point where both the Lipschitz continuity of $m(x)$ and the choice of the phase space $D\left(A^{3 / 4}\right) \times D\left(A^{1 / 4}\right)$ come into play.

When the coefficient $c(t)$ is not Lipschitz continuous, solutions to (1.6) can exhibit a severe derivative loss for all positive times, as shown in the seminal paper [8]. In the sequel we call (DGCS)-phenomenon this instantaneous loss of regularity. As a consequence, when $c(t)$ is not Lipschitz continuous the initial value problem for (1.6) is well-posed only for very regular initial data, such as Gevrey or analytic functions. We refer to [8] for the linear theory, and to [14] for its application to Kirchhoff equations.

The existence results for linear equations and the (DGCS)-phenomenon motivate the three main assumptions in the theory of Kirchhoff equations, namely Lipschitz continuity of the nonlinearity $m(x)$, strict hyperbolicity, and initial data at least in $D\left(A^{3 / 4}\right) \times D\left(A^{1 / 4}\right)$. These three main assumptions become three insurmountable barriers when trying to extend the theory.

Kirchhoff equations with standard damping Equation (1.1) with $\delta>0$ and $\sigma=0$ has long been studied starting from the 80 s, both in the strictly hyperbolic case (see $[12,38]$ ), and in the mildly degenerate case (see [33] for nonlinear terms of the form $m(x)=x^{\alpha}$ and [13] for general Lipschitz continuous nonlinear terms). 
As for local solutions, the state of the art is exactly the same as in the non-dissipative case. As for global solutions, it was possible to prove that they exist in Sobolev spaces (such as $D(A) \times D\left(A^{1 / 2}\right)$ and sometimes $D\left(A^{3 / 4}\right) \times D\left(A^{1 / 4}\right)$ ) provided that initial data are small enough in the same space and $m(x)$ is Lipschitz continuous. The interested reader is referred to the survey [16] for further details.

In other words, a standard damping seems to be impotent against the classical three barriers. As a further evidence, it was recently shown in [19] that the linearized equation with standard dissipation

$$
u^{\prime \prime}(t)+2 \delta u^{\prime}(t)+c(t) A u(t)=0
$$

can exhibit the (DGCS)-phenomenon if the coefficient $c(t)$ is not Lipschitz continuous.

Equations with strong damping Mathematical models with strong damping were proposed in [4], leading to the linear equation

$$
u^{\prime \prime}(t)+2 \delta A^{\sigma} u^{\prime}(t)+A u(t)=0,
$$

which afterwards was rigorously analyzed by many authors from different points of view, and with different choices of $\sigma$. Here we just quote the first papers $[5,6,7]$ (see also the more recent [22]) devoted to analyticity properties of the semigroup associated to (1.8), and the papers $[24,25,37]$ by the dispersive school where (1.8) is considered in the concrete case where $A u=-\Delta u$ in $\mathbb{R}^{n}$ or special classes of unbounded domains. Finally, equation (1.8) was considered in [20] in full generality, namely for every $\sigma \geq 0$ and every nonnegative self-adjoint operator $A$.

Concerning Kirchhoff equations with strong dissipation, the first result we are aware of was obtained by Nishihara in [31]. He considered the case $\sigma=1$, and proved global existence for initial data in $D(A) \times D\left(A^{1 / 2}\right)$ provided that the nonlinearity is Lipschitz continuous and satisfies the degenerate hyperbolicity condition (1.5). Some years later, Matos and Pereira [28] improved this result by showing global existence in the energy space $D\left(A^{1 / 2}\right) \times H$ with the same assumptions on $\sigma$ and $m(x)$. In other words, in the case $\sigma=1$ Nishihara overcame one of the three classical barriers (strict hyperbolicity), and then Matos and Pereira overcame one more barrier (regularity of initial data). Both results do not extend to $\sigma<1$, and seem to exploit the Lipschitz continuity of $m(x)$ in an essential way. We discuss this technical point at the beginning of section 6 .

As far as we know, references [31] and [28] represented the state of the art up to now, and all subsequent papers on the subject were devoted just to qualitative properties of these solutions, such as decay estimates (see for example [32, 34]).

In this paper we consider equation (1.1) in the full range $\sigma>0$. Two different regimes appear.

- When $\sigma>1 / 2$ (supercritical regime) the dissipation prevails in an overwhelming way. Indeed, in Theorem 2.1 we prove global existence in the energy space assuming only continuity and degenerate hyperbolicity of the nonlinear term. 
- When $0<\sigma \leq 1 / 2$ (subcritical regime) the dissipation competes with the continuity modulus of the nonlinear term. In Theorem 2.2 we assume strict hyperbolicity, and we prove

- local existence for all initial data in a phase space depending on $\sigma$ and on the continuity modulus of $m$.

- global existence provided that initial data are small in the same space.

Just to give an example, if $m(x)$ is $\beta$-Hölder continuous for some $\beta \in(1-2 \sigma, 1)$, then we obtain local/global existence in phase spaces of the form $D\left(A^{\alpha+1 / 2}\right) \times$ $D\left(A^{\alpha}\right)$ with

$$
4 \alpha \beta \geq 1-2 \sigma
$$

Note that this condition allows nonlinear terms which are not Lipschitz continuous and initial data with $\alpha<1 / 4$.

Overview of the technique Theorem 2.1 and Theorem 2.2 can be proved in many different ways (here we use a fixed point argument on the coefficient $c(t)$, but also fixed point arguments on $u(t)$ or Galerkin approximations could work) after that three main tools have been developed.

The first tool is linearization. The results for the non-dissipative Kirchhoff equation (1.3) are based on the theory of the linearized equation (1.6). In the same way, our results for (1.1) rely on the theory of the linear equation

$$
u^{\prime \prime}(t)+2 \delta A^{\sigma} u^{\prime}(t)+c(t) A u(t)=0
$$

where $c(t)$ is a time-dependent coefficient. This theory was developed in the recent paper [19]. The following two regimes appeared.

- In the supercritical regime $\sigma>1 / 2$ the dissipation prevails, and (1.10) generates a semigroup in the energy space $D\left(A^{1 / 2}\right) \times H$ provided that $c(t)$ is measurable and satisfies the degenerate hyperbolicity assumption

$$
0 \leq c(t) \leq \mu_{2}
$$

- In the subcritical regime $0<\sigma \leq 1 / 2$, if the strict hyperbolicity assumption (1.7) is satisfied, the dissipation competes with the continuity modulus of $c(t)$. For example, if $c(t)$ is $\gamma$-Hölder continuous for some $\gamma \in(0,1)$, then (1.10) generates a semigroup in the energy space if $\gamma>1-2 \sigma$, while (1.10) can exhibit the (DGCS)phenomenon if $\gamma<1-2 \sigma$.

Let us consider for example the subcritical regime. In the non-dissipative case, or in the case with standard dissipation, the key point was controlling the Lipschitz constant of $c(t)$. Now the linear theory tells us that it is enough to control the $\gamma$-Hölder constant 
of $c(t)$ for some $\gamma>1-2 \sigma$. In turn, this constant depends on the continuity moduli of $m(x)$ and $\left|A^{1 / 2} u(t)\right|^{2}$.

In order to control the latter, we develop our second tool. We prove that, when (1.10) generates a continuous semigroup, the continuity modulus of $\left|A^{1 / 2} u(t)\right|^{2}$ depends on initial data only (and not on $c(t)$ ), according to the following scheme:

$$
\begin{aligned}
\left(u_{0}, u_{1}\right) \in D\left(A^{1 / 2}\right) \times H & \rightsquigarrow\left|A^{1 / 2} u(t)\right|^{2} \text { is continuous, } \\
\left(u_{0}, u_{1}\right) \in D\left(A^{3 / 4}\right) \times D\left(A^{1 / 4}\right) & \rightsquigarrow\left|A^{1 / 2} u(t)\right|^{2} \text { is Lipschitz continuous, } \\
\left(u_{0}, u_{1}\right) \in D\left(A^{\alpha+1 / 2}\right) \times D\left(A^{\alpha}\right) & \rightsquigarrow\left|A^{1 / 2} u(t)\right|^{2} \text { is } 4 \alpha \text {-Hölder continuous. }
\end{aligned}
$$

Thus, if the nonlinear term is $\beta$-Hölder continuous for some $\beta \in(0,1)$, and initial data are in $D\left(A^{\alpha+1 / 2}\right) \times D\left(A^{\alpha}\right)$ for some $\alpha \in(0,1 / 4)$, then $c(t)$ is Hölder continuous with exponent $4 \alpha \beta$, and when $4 \alpha \beta>1-2 \sigma$ the linear theory is well-posed. This is where condition (1.9) appears.

The last tool we develop allows to deal with the equality case $4 \alpha \beta=1-2 \sigma$. Roughly speaking, the reason is that an initial condition which is "purely" in $D\left(A^{\alpha+1 / 2}\right) \times D\left(A^{\alpha}\right)$ does not exist, in the same way as a vector which is just in $H$, and nothing more, does not exist. Indeed, any vector in $H$ lies actually in some better space, and this better space guarantees a better continuity modulus for $\left|A^{1 / 2} u(t)\right|^{2}$. We state this point formally in Proposition 5.6.

Conclusions Our results are the best possible ones in the supercritical regime, where all three barriers have been overcome to the maximum extent. In the subcritical regime we extended the theory beyond two of the three barriers, namely to less regular nonlinearities and less regular initial data. The width of this extension depends on $\sigma$ and it is the best possible one according to the linear theory, because the linearized equation does admit the (DGCS)-phenomenon beyond the spaces we considered in this paper.

Our existence results confirm the general paradigm according to which Kirchhoff equations are well-posed, at least locally in time, whenever the (DGCS)-phenomenon is excluded. What happens beyond remains an open problem, and deep new ideas are likely to be needed (see [14] for a partial result).

We also hope that these results could give an indication about the regularizing effects one can expect when adding a strong dissipation to quasilinear hyperbolic equations.

Structure of the paper This paper is organized as follows. In section 2 we introduce the functional setting and we state our main results concerning local and global solvability for problem (1.1)-(1.2). In section 3 we recall the results we need from the linear theory developed in [19]. In section 4 we study how solutions to the linear problem (1.10) depend continuously on the coefficient $c(t)$. In section 5 we introduce our interpolation spaces. In section 6 we prove a priori estimates for Kirchhoff equations after discussing what goes wrong in the classical ones. Finally, in section 7 we prove our main results, actually in a stronger form based on our interpolation spaces. 


\section{Notation and statement of main results}

Functional spaces Let $H$ be a separable Hilbert space. Let us assume that $H$ admits a countable complete orthonormal system $\left\{e_{k}\right\}_{k \in \mathbb{N}}$ made by eigenvectors of $A$. We denote the corresponding eigenvalues by $\lambda_{k}^{2}$ (with the agreement that $\lambda_{k} \geq 0$ ), so that $A e_{k}=\lambda_{k}^{2} e_{k}$ for every $k \in \mathbb{N}$. We do not assume the monotonicity of the sequence $\left\{\lambda_{k}\right\}$, even if this assumption would be reasonable in many applications. Every $u \in H$ can be written in a unique way in the form $u=\sum_{k=0}^{\infty} u_{k} e_{k}$, where $u_{k}=\left\langle u, e_{k}\right\rangle$ are the Fourier components of $u$. In other words, the Hilbert space $H$ can be identified with the set of sequences $\left\{u_{k}\right\}$ of real numbers such that $\sum_{k=0}^{\infty} u_{k}^{2}<+\infty$.

We stress that this is just a simplifying assumption, with substantially no loss of generality. Indeed, according to the spectral theorem in its general form (see for example Theorem VIII.4 in [36]), one can always identify $H$ with $L^{2}(M, \mu)$ for a suitable measure space $(M, \mu)$, in such a way that under this identification the operator $A$ acts as a multiplication operator by some measurable function $\lambda^{2}(\xi)$. All definitions and statements in the sequel can be easily extended to the general setting just by replacing the sequence $\left\{\lambda_{k}\right\}$ with the function $\lambda(\xi)$, and the sequence $\left\{u_{k}\right\}$ of Fourier components of $u$ with the element $\widehat{u}(\xi)$ of $L^{2}(M, \mu)$ corresponding to $u$ under the identification of $H$ with $L^{2}(M, \mu)$.

Powers of the operator $A$ are just defined as $A^{\alpha} u:=\sum_{k=0}^{\infty} \lambda_{k}^{2 \alpha} u_{k} e_{k}$, where of course

$$
u \in D\left(A^{\alpha}\right) \Longleftrightarrow \sum_{k=0}^{\infty}\left(1+\lambda_{k}^{4 \alpha}\right) u_{k}^{2}<+\infty .
$$

It is well-known that $D\left(A^{\alpha}\right)$ is itself a Hilbert space, with norm defined by

$$
|u|_{D\left(A^{\alpha}\right)}^{2}:=|u|^{2}+\left|A^{\alpha} u\right|^{2} .
$$

In concrete applications, $H$ is the space $L^{2}(\Omega)$ for some open set $\Omega \subseteq \mathbb{R}^{n}$, and $A u=-\Delta u$, with suitable boundary conditions. If the boundary of $\Omega$ is regular enough, $D\left(A^{\alpha}\right)$ is the set of functions in the Sobolev space $H^{2 \alpha}(\Omega)$ attaining the boundary conditions in a suitable sense.

Continuity moduli Throughout this paper we call continuity modulus any continuous function $\omega:[0,+\infty) \rightarrow[0,+\infty)$ such that $\omega(0)=0, \omega(x)>0$ for every $x>0$, and moreover

$$
\begin{aligned}
& x \rightarrow \omega(x) \text { is a nondecreasing function, } \\
& x \rightarrow \frac{x}{\omega(x)} \text { is a nondecreasing function. }
\end{aligned}
$$

From (2.1) and (2.2) one can easily deduce that every continuity modulus satisfies

$$
\omega(L x) \leq \max \{1, L\} \omega(x) \quad \forall x \geq 0, \quad \forall L \geq 0,
$$


and that the composition of two continuity moduli is again a continuity modulus. We exploit these two properties several times in the sequel.

A function $c:[0,+\infty) \rightarrow \mathbb{R}$ is said to be $\omega$-continuous if

$$
|c(a)-c(b)| \leq \omega(|a-b|) \quad \forall a \geq 0, \forall b \geq 0 .
$$

More generally, a function $c: X \rightarrow \mathbb{R}$ (with $X \subseteq \mathbb{R}$ ) is said to be $\omega$-continuous if it satisfies the same inequality for every $a$ and $b$ in $X$.

Main results The main results of this paper concern local and global existence for problem (1.1)-(1.2). The first one gives a full answer in the case $\sigma>1 / 2$, which we call supercritical dissipation.

Theorem 2.1 (Supercritical dissipation). Let us consider problem (1.1)-(1.2) under the following assumptions:

- A is a self-adjoint nonnegative operator on a separable Hilbert space $H$,

- $m:[0,+\infty) \rightarrow[0,+\infty)$ is a continuous function,

- $\sigma>1 / 2$ and $\delta>0$ are two real numbers,

- $\left(u_{0}, u_{1}\right) \in D\left(A^{1 / 2}\right) \times H$.

Then the problem admits at least one global solution

$$
u \in C^{0}\left([0,+\infty), D\left(A^{1 / 2}\right)\right) \cap C^{1}([0,+\infty), H) .
$$

Our second result addresses the case $\sigma \leq 1 / 2$, which we call subcritical dissipation.

Theorem 2.2 (Subcritical dissipation). Let us consider problem (1.1)-(1.2) under the following assumptions:

- A is a self-adjoint nonnegative operator on a separable Hilbert space $H$,

- $m:[0,+\infty) \rightarrow[0,+\infty)$ satisfies the strict hyperbolicity assumption (1.4) and the $\omega$-continuity assumption (2.4) for some continuity modulus $\omega_{m}(x)$,

- $\sigma \in(0,1 / 2]$ and $\delta>0$ are two real numbers,

- there exists $\alpha \in[0,1 / 4)$ such that

$$
\limsup _{x \rightarrow 0^{+}} \frac{\left[\omega_{m}(x)\right]^{4 \alpha}}{x^{1-2 \sigma}}<+\infty
$$

- $\left(u_{0}, u_{1}\right) \in D\left(A^{\alpha+1 / 2}\right) \times D\left(A^{\alpha}\right)$ for the same $\alpha \in[0,1 / 4)$ which appears in (2.5).

Then the following conclusions hold true. 
(1) (Local existence) There exists $T>0$ such that problem (1.1)-(1.2) admits at least one local solution

$$
u \in C^{0}\left([0, T], D\left(A^{\alpha+1 / 2}\right)\right) \cap C^{1}\left([0, T], D\left(A^{\alpha}\right)\right) .
$$

(2) (Global existence) There exists $\varepsilon_{0}>0$ such that, if initial conditions satisfy

$$
\left|u_{1}\right|^{2}+\left|A^{1 / 2} u_{0}\right|^{2}+\left|A^{\alpha} u_{1}\right|^{2}+\left|A^{\alpha+1 / 2} u_{0}\right|^{2} \leq \varepsilon_{0},
$$

then there exists at least one global solution

$$
u \in C^{0}\left([0,+\infty), D\left(A^{\alpha+1 / 2}\right)\right) \cap C^{1}\left([0,+\infty), D\left(A^{\alpha}\right)\right) .
$$

Remark 2.3. In Theorem 2.2 , the $\omega$-continuity assumption on $m(x)$ can be easily weakened to local $\omega$-continuity, meaning that we can limit ourselves to assume that for every $R>0$ the function $m(x)$ has continuity modulus $\omega_{R}(x)$ in $[0, R]$, and all these continuity moduli satisfy (2.5) with the same exponent $\alpha$ (of course the limsup may depend on $R$ in an arbitrary way). We spare the reader from this standard generalization.

Remark 2.4. As a simple example of application of Theorem 2.2, let us consider the case where $m(x)$ is $\beta$-Hölder continuous, namely $\omega_{m}(x)=M x^{\beta}$ for some constants $M>0$ and $\beta \in(0,1)$.

If $\beta \in(1-2 \sigma, 1)$, then $(2.5)$ is satisfied if we take $\alpha:=(1-2 \sigma) /(4 \beta)$. This exponent is less than $1 / 4$, so that we obtain solvability beyond two of the classical three barriers.

If $\beta \in(0,1-2 \sigma)$, then there is no $\alpha \leq 1 / 4$ for which (2.5) is satisfied, so that our theory does not apply to this case.

In the limit case $\beta=1-2 \sigma$, one should take $\alpha=1 / 4$ in order to fulfil (2.5). This case in not included in Theorem 2.2 above. Nevertheless, a careful inspection of the proof reveals that in this limit case one can obtain both local and global solvability in $D\left(A^{3 / 4}\right) \times D\left(A^{1 / 4}\right)$ provided that initial data are small enough (so that the smallness is required also for local existence). We spare the reader from this subtlety.

Remark 2.5. The following pictures provide a rough description of the state of the art. In the horizontal axis we represent the regularity of $m(x)$. With some abuse of notation, values $\beta \in(0,1)$ mean that $m(x)$ is $\beta$-Hölder continuous, $\beta=1$ means that it is Lipschitz continuous, $\beta>1$ means even more regular. The value $\alpha$ in the vertical axis represents the phase space $D\left(A^{\alpha+1 / 2}\right) \times D\left(A^{\alpha}\right)$.

In the case $\sigma=0$ (not addressed in this paper), both local existence and global existence for small data are known in the region where $\alpha \geq 1 / 4$ and $\beta \geq 1$.

In the case $0<\sigma<1 / 2$, Theorem 2.2 gives the same conclusions in a larger region, which is bounded by the line $\alpha=(1-2 \sigma) / 4$, the line $\beta=1-2 \sigma$, and the curve $\alpha=(1-2 \sigma) /(4 \beta)$. The vertical part of the boundary is not included, or at least it is just partially included as explained at the end of Remark 2.4. 
Strict hyperbolicity, local existence for all data, global existence for small data

Degenerate hyperbolicity, global existence for all data
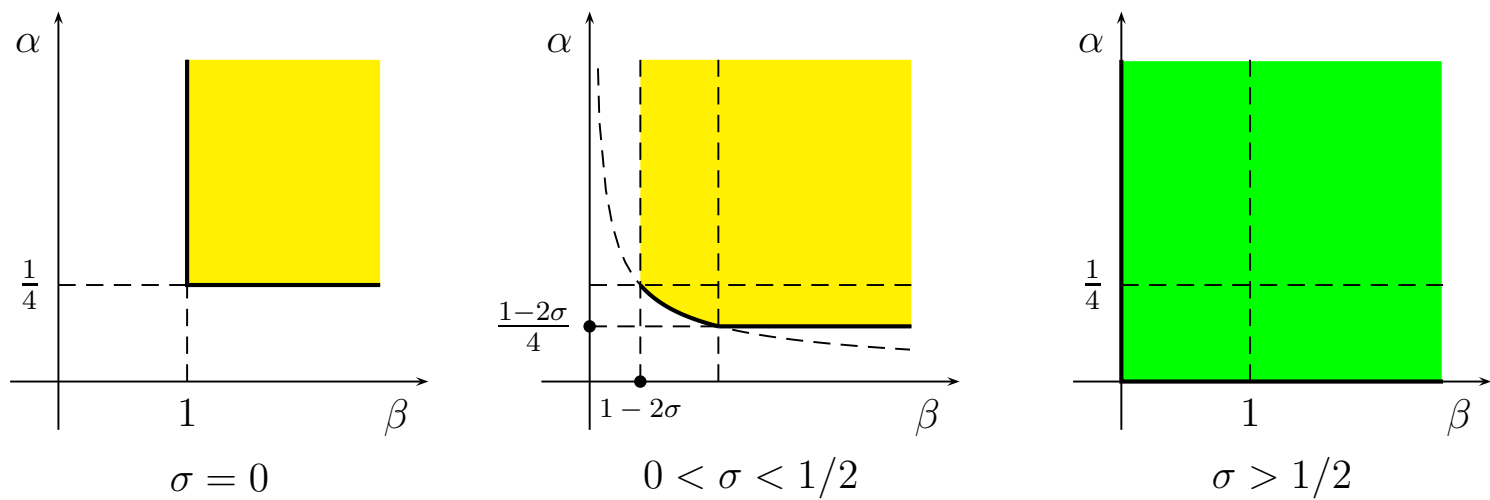

In the case $\sigma>1 / 2$, Theorem 2.1 guarantees global existence in the phase space, independently of the size of initial data, even in the degenerate hyperbolic case.

Remark 2.6. Let us discuss in detail the borderline case $\sigma=1 / 2$. First of all, it fits in the framework of Theorem 2.2, where for $\sigma=1 / 2$ assumption (2.5) holds true for free, even if $\alpha=0$. Thus we obtain both local existence and global existence for small data in the energy space $D\left(A^{1 / 2}\right) \times H$, provided that $m(x)$ is continuous and satisfies the strict hyperbolicity condition (1.4).

Concerning the degenerate hyperbolic case, the limit exponent $\sigma=1 / 2$ is not included in Theorem 2.1 as stated above. Nevertheless, a careful inspection of the proof reveals that some conclusions hold true also when $\sigma=1 / 2$. Indeed, we can prove that a local solution exists for all initial data $\left(u_{0}, u_{1}\right)$ in the energy space such that $4 \delta^{2}>m\left(\left|A^{1 / 2} u_{0}\right|^{2}\right)$, and this solution survives as long as $4 \delta^{2}>m\left(\left|A^{1 / 2} u(t)\right|^{2}\right)$. This could be always the case for suitable choices of $m(x)$ and $\left(u_{0}, u_{1}\right)$, for example in the trivial case where $m(x)<4 \delta^{2}$ for every $x \geq 0$. We spare the reader from this further subtlety.

This discussion shows that in the critical case $\sigma=1 / 2$ we have coexistence of two statements, one dealing with strictly hyperbolic nonlinearities and one dealing with degenerate hyperbolic nonlinearities. This coexistence reflects the analogous coexistence in the linear case (see [19, Remark 3.7]) and suggests the existence of a more general unifying statement, which could probably deserve further investigation.

Remark 2.7. Solutions to (1.1) are also solutions to (1.10), hence they inherit all properties of solutions to linear equations. In particular, multiple choices of the phase space are possible when $\sigma>1 / 2$, as observed in $[20,19]$. Therefore, under the assumptions of Theorem 2.1, the problem is well-posed not only in the energy space or more generally in $D\left(A^{\alpha+1 / 2}\right) \times D\left(A^{\alpha}\right)$, but also in all phase spaces of the form $D\left(A^{\alpha}\right) \times D\left(A^{\beta}\right)$ with $\alpha \geq 1 / 2, \beta \geq 0$, and $1-\sigma \leq \alpha-\beta \leq \sigma$. 


\section{The linearized equation - Previous results}

In this section we collect all the results we need from the linear theory developed in [19]. To this end, we introduce some further notation. Given any $\nu \geq 0$, we write $H$ as an orthogonal direct sum

$$
H:=H_{\nu,-} \oplus H_{\nu,+},
$$

where $H_{\nu,-}$ is the closure of the subspace generated by all eigenvectors of $A$ relative to eigenvalues $\lambda_{k}<\nu$, and $H_{\nu,+}$ is the closure of the subspace generated by all eigenvectors of $A$ relative to eigenvalues $\lambda_{k} \geq \nu$. For every vector $u \in H$, we write $u_{\nu,-}$ and $u_{\nu,+}$ to denote its components with respect to the decomposition (3.1). We point out that $H_{\nu,-}$ and $H_{\nu,+}$ are $A$-invariant subspaces of $H$, and that $A$ is a bounded operator when restricted to $H_{\nu,-}$, and a coercive operator when restricted to $H_{\nu,+}$ if $\nu>0$.

Here we state the results in local form, namely with coefficients $c(t)$ defined in some time-interval $[0, T]$. They follow immediately from the corresponding results proved in [19] for coefficients defined in $[0,+\infty$ ) (it is enough to extend the coefficient by setting $c(t)=c(T)$ for every $t \geq T)$.

\subsection{Existence and estimates in the energy space}

As always, we distinguish the supercritical and the subcritical case. In both cases, we limit ourselves to initial data in the energy space.

Theorem A (Supercritical dissipation, see [19, Theorem 3.1]). Let $T>0$, and let us consider problem (1.10)-(1.2) under the following assumptions:

- $A$ is a self-adjoint nonnegative operator on a separable Hilbert space $H$,

- the coefficient $c:[0, T] \rightarrow \mathbb{R}$ is measurable and satisfies the degenerate hyperbolicity assumption (1.11),

- $\sigma$ and $\delta$ are two positive real numbers such that either $\sigma>1 / 2$, or $\sigma=1 / 2$ and $4 \delta^{2} \geq \mu_{2}$,

- $\left(u_{0}, u_{1}\right) \in D\left(A^{1 / 2}\right) \times H$.

Then the problem has a unique solution

$$
u \in C^{0}\left([0, T], D\left(A^{1 / 2}\right)\right) \cap C^{1}([0, T], H) .
$$

Moreover, there exists a constant $K_{1}\left(\delta, \mu_{2}\right)$, depending only on $\delta$ and $\mu_{2}$ (and in particular independent of $T$ ), with the following property. For every $\nu \geq 1$ such that

$$
4 \delta^{2} \nu^{4 \sigma-2} \geq \mu_{2}
$$

it turns out that

$$
\left|u^{\prime}(t)\right|^{2}+\left|A^{1 / 2} u(t)\right|^{2} \leq K_{1}\left(\delta, \mu_{2}\right) e^{\nu\left(1+\mu_{2}\right) t}\left(\left|u_{1}\right|^{2}+\left|A^{1 / 2} u_{0}\right|^{2}\right) \quad \forall t \in[0, T],
$$


and more precisely for every $t \in[0, T]$ it turns out that

$$
\begin{gathered}
\left|u_{\nu,-}^{\prime}(t)\right|^{2}+\left|A^{1 / 2} u_{\nu,-}(t)\right|^{2} \leq e^{\nu\left(1+\mu_{2}\right) t}\left(\left|u_{1, \nu,-}\right|^{2}+\left|A^{1 / 2} u_{0, \nu,-}\right|^{2}\right), \\
\left|u_{\nu,+}^{\prime}(t)\right|^{2}+\left|A^{1 / 2} u_{\nu,+}(t)\right|^{2} \leq K_{1}\left(\delta, \mu_{2}\right)\left(\left|u_{1, \nu,+}\right|^{2}+\left|A^{1 / 2} u_{0, \nu,+}\right|^{2}\right) .
\end{gathered}
$$

Theorem B (Subcritical dissipation, see [19, Theorem 3.2]). Let $T>0$, and let us consider problem (1.10)-(1.2) under the following assumptions:

- A is a self-adjoint nonnegative operator on a separable Hilbert space $H$,

- the coefficient $c:[0, T] \rightarrow \mathbb{R}$ satisfies the strict hyperbolicity assumption (1.7) and the $\omega$-continuity assumption (2.4) for some continuity modulus $\omega(x)$,

- $\sigma \in[0,1 / 2]$ and $\delta>0$ are two real numbers such that

$$
4 \delta^{2} \mu_{1}>\Lambda_{\infty}^{2}+2 \delta \Lambda_{\infty}
$$

where we set

$$
\Lambda_{\infty}:=\limsup _{\varepsilon \rightarrow 0^{+}} \frac{\omega(\varepsilon)}{\varepsilon^{1-2 \sigma}},
$$

- $\left(u_{0}, u_{1}\right) \in D\left(A^{1 / 2}\right) \times H$.

Then the problem has a unique solution with the regularity stated in (3.2).

Moreover, there exists a constant $K_{2}\left(\delta, \mu_{1}, \mu_{2}\right)$, depending only on $\delta, \mu_{1}$ and $\mu_{2}$ (and in particular independent of $T$ ), with the following property. For every $\nu \geq 1$ such that

$$
4 \delta^{2} \mu_{1} \geq\left[\lambda^{1-2 \sigma} \omega\left(\frac{1}{\lambda}\right)\right]^{2}+2 \delta\left[\lambda^{1-2 \sigma} \omega\left(\frac{1}{\lambda}\right)\right] \quad \forall \lambda \geq \nu
$$

it turns out that

$$
\left|u^{\prime}(t)\right|^{2}+\left|A^{1 / 2} u(t)\right|^{2} \leq K_{2}\left(\delta, \mu_{1}, \mu_{2}\right) e^{\nu\left(1+\mu_{2}\right) t}\left(\left|u_{1}\right|^{2}+\left|A^{1 / 2} u_{0}\right|^{2}\right) \quad \forall t \in[0, T],
$$

and more precisely for every $t \in[0, T]$ it turns out that

$$
\begin{array}{r}
\left|u_{\nu,-}^{\prime}(t)\right|^{2}+\left|A^{1 / 2} u_{\nu,-}(t)\right|^{2} \leq e^{\nu\left(1+\mu_{2}\right) t}\left(\left|u_{1, \nu,-}\right|^{2}+\left|A^{1 / 2} u_{0, \nu,-}\right|^{2}\right), \\
\left|u_{\nu,+}^{\prime}(t)\right|^{2}+\left|A^{1 / 2} u_{\nu,+}(t)\right|^{2} \leq K_{2}\left(\delta, \mu_{1}, \mu_{2}\right)\left(\left|u_{1, \nu,+}\right|^{2}+\left|A^{1 / 2} u_{0, \nu,+}\right|^{2}\right) .
\end{array}
$$

The key tool in the proof of Theorem A and Theorem B are some estimates on the Fourier components of the solution. We recall these estimates because we need them in the sequel. Let $\left\{u_{k}(t)\right\}$ denote the components of $u(t)$ with respect to the usual orthonormal system $\left\{e_{k}\right\}$, and let $\left\{u_{0 k}\right\}$ and $\left\{u_{1 k}\right\}$ denote the corresponding components of initial conditions. It turns out that $u_{k}(t)$ is a solution to

$$
u_{k}^{\prime \prime}(t)+2 \delta \lambda_{k}^{2 \sigma} u_{k}^{\prime}(t)+\lambda_{k}^{2} c(t) u_{k}(t)=0,
$$


with initial data

$$
u_{k}(0)=u_{0 k}, \quad u_{k}^{\prime}(0)=u_{1 k} .
$$

Estimates on low-frequency components are quite general (see [19, Remark 3.3]). Let us assume only that $c(t)$ is a measurable function satisfying the degenerate hyperbolicity assumption (1.11) in $[0, T]$, and that $\delta \geq 0, \sigma \geq 0, \nu \geq 0$ and $\lambda_{k} \leq \nu$. Then it turns out that

$$
\left|u_{k}^{\prime}(t)\right|^{2}+\lambda_{k}^{2}\left|u_{k}(t)\right|^{2} \leq e^{\nu\left(1+\mu_{2}\right) t}\left(\left|u_{1 k}\right|^{2}+\lambda_{k}^{2}\left|u_{0 k}\right|^{2}\right) \quad \forall t \in[0, T]
$$

Summing over all indices $k$ with $\lambda_{k}<\nu$ we obtain both (3.5) and (3.10).

Estimates on high-frequency components are more delicate, and different in the two cases. In the supercritical case, let us assume that $c(t), \delta$ and $\sigma$ are as in Theorem A, that $\nu \geq 1$ satisfies (3.3), and that $\lambda_{k} \geq \nu$. Then it turns out that

$$
\left|u_{k}^{\prime}(t)\right|^{2}+\lambda_{k}^{2}\left|u_{k}(t)\right|^{2} \leq K_{1}\left(\delta, \mu_{2}\right)\left(\left|u_{1 k}\right|^{2}+\lambda_{k}^{2}\left|u_{0 k}\right|^{2}\right) \quad \forall t \in[0, T],
$$

where the constant $K_{1}\left(\delta, \mu_{2}\right)$ depends only on $\delta$ and $\mu_{2}$. This was established in [19, Lemma 5.1]. Since the constant is independent of $k$, summing over all indices $k$ with $\lambda_{k} \geq \nu$ we obtain (3.6). Finally, (3.4) follows from (3.5) and (3.6) (in this point we need that $K_{1}\left(\delta, \mu_{2}\right) \geq 1$, which in turn is a consequence of (3.6) with $\left.t=0\right)$.

In the subcritical case, let us assume that $c(t), \delta$ and $\sigma$ are as in Theorem $\mathrm{B}$, that $\nu \geq 1$ satisfies (3.9), and that $\lambda_{k} \geq \nu$. Then it turns out that

$$
\left|u_{k}^{\prime}(t)\right|^{2}+\lambda_{k}^{2}\left|u_{k}(t)\right|^{2} \leq K_{2}\left(\delta, \mu_{1}, \mu_{2}\right)\left(\left|u_{1 k}\right|^{2}+\lambda_{k}^{2}\left|u_{0 k}\right|^{2}\right) \quad \forall t \in[0, T],
$$

where the constant $K_{2}\left(\delta, \mu_{1}, \mu_{2}\right)$ depends only on $\delta, \mu_{1}$ and $\mu_{2}$. This was established in [19, Lemma 5.2]. Since the constant is independent of $k$, summing over all indices $k$ with $\lambda_{k} \geq \nu$ we obtain (3.11).

\subsection{The regularizing effect}

The strong dissipation has a regularizing effect in the range $\sigma \in(0,1)$. This effect was studied in [19] in terms of Gevrey spaces. Here we just state what we need in the sequel, without relying on the theory of abstract Gevrey spaces.

Let us start with the supercritical case.

Theorem C (Supercritical dissipation - Regularizing effect). Let $u(t)$ be a solution to problem (1.10)-(1.2) under the same assumptions of Theorem A. Let us assume in addition that either $\sigma \in(1 / 2,1)$, or $\sigma=1 / 2$ and $4 \delta^{2}>\mu_{2}$.

Let us consider the function

$$
C(t):=\int_{0}^{t} c(s) d s \quad \forall t \in[0, T],
$$

and let us distinguish three cases. 
(1) If $C(t)>0$ for every $t \in(0, T]$, then

$$
u \in C^{1}\left((0, T], D\left(A^{\alpha}\right)\right) \quad \forall \alpha \geq 0 .
$$

(2) If $C(t)=0$ for every $t \in[0, T]$, then

$$
u^{\prime} \in C^{\infty}\left((0, T], D\left(A^{\alpha}\right)\right) \quad \forall \alpha \geq 0 .
$$

(3) If there exists $S_{0} \in(0, T)$ such that $C(t)=0$ for every $t \in\left[0, S_{0}\right]$, and $C(t)>0$ for every $t \in\left(S_{0}, T\right]$, then

$$
u \in C^{1}\left(\left(S_{0}, T\right], D\left(A^{\alpha}\right)\right) \quad \forall \alpha \geq 0,
$$

and

$$
u^{\prime} \in C^{0}\left(\left(0, S_{0}\right) \cup\left(S_{0}, T\right], D\left(A^{\alpha}\right)\right) \quad \forall \alpha \geq 0 .
$$

In the first case the regularity (3.15) follows from [19, Theorem 3.8].

In the second case it turns out that also $c(t)=0$ for every $t \in[0, T]$, so that $u^{\prime}(t)$ is the solution to the parabolic problem

$$
v^{\prime}(t)+2 \delta A^{\sigma} v(t)=0, \quad v(0)=u_{1},
$$

and therefore the regularity (3.16) follows from the regularizing effect of parabolic problems.

In the third case the regularity in $\left(S_{0}, T\right]$ follows from [19, Theorem 3.8] as in the first case, while the regularity of $u^{\prime}(t)$ in $\left(0, S_{0}\right)$ follows from the parabolic problem as in the second case (indeed $c(t)=0$ for every $t \in\left[0, S_{0}\right]$ ).

The last result concerns the regularizing effect in the subcritical case, and it is an immediate consequence of [19, Theorem 3.9].

Theorem D (Subcritical dissipation - Regularizing effect). Let $u(t)$ be a solution to problem (1.10)-(1.2) under the same assumptions of Theorem B. Let us assume in addition that $\sigma \in(0,1 / 2]$, so that the case $\sigma=0$ is excluded.

Then (3.15) holds true.

\section{Passing to the limit in linear problems}

In this section we consider a sequence of linear problems

$$
u_{n}^{\prime \prime}(t)+2 \delta A^{\sigma} u_{n}^{\prime}(t)+c_{n}(t) A u_{n}(t)=0,
$$

with fixed initial data

$$
u_{n}(0)=u_{0}, \quad u_{n}^{\prime}(0)=u_{1}
$$


We assume that the sequence of coefficients $c_{n}:[0, T] \rightarrow[0,+\infty)$ pointwise converges to a limit coefficient $c_{\infty}:[0, T] \rightarrow[0,+\infty)$, namely

$$
\lim _{n \rightarrow+\infty} c_{n}(t)=c_{\infty}(t) \quad \forall t \in[0, T] .
$$

We investigate the convergence of solutions to (4.1)-(4.2) to the solution of the limit problem

$$
\begin{gathered}
u_{\infty}^{\prime \prime}(t)+2 \delta A^{\sigma} u_{\infty}^{\prime}(t)+c_{\infty}(t) A u_{\infty}(t)=0, \\
u_{\infty}(0)=u_{0}, \quad u_{\infty}^{\prime}(0)=u_{1} .
\end{gathered}
$$

Our goal is proving uniform convergence of solutions in the energy space, namely

$$
\lim _{n \rightarrow+\infty} \sup _{t \in[0, T]}\left(\left|u_{\infty}^{\prime}(t)-u_{n}^{\prime}(t)\right|^{2}+\left|A^{1 / 2}\left(u_{\infty}(t)-u_{n}(t)\right)\right|^{2}\right)=0 .
$$

We obtain the following two results, which are probably interesting in themselves.

Theorem 4.1 (Convergence in the supercritical case). Let $u_{n}(t)$ be the sequence of solutions to the approximating problems (4.1)-(4.2), and let $u_{\infty}(t)$ be the solution to the limit problem (4.4)-(4.5).

Let us assume that

- A is a self-adjoint nonnegative operator on a separable Hilbert space $H$,

- the coefficients $c_{n}:[0, T] \rightarrow \mathbb{R}$ are measurable and satisfy the degenerate hyperbolicity assumption (1.11) with the same $\mu_{2}$,

- $c_{n}(t)$ pointwise converges to $c_{\infty}(t)$,

- $\sigma$ and $\delta$ are two positive real numbers such that either $\sigma>1 / 2$, or $\sigma=1 / 2$ and $4 \delta^{2} \geq \mu_{2}$

- $\left(u_{0}, u_{1}\right) \in D\left(A^{1 / 2}\right) \times H$.

Then $u_{n} \rightarrow u_{\infty}$ in the sense of (4.6).

Theorem 4.2 (Convergence in the subcritical case). Let $u_{n}(t)$ be the sequence of solutions to the approximating problems (4.1)-(4.2), and let $u_{\infty}(t)$ be the solution to the limit problem (4.4)-(4.5).

Let us assume that

- $A$ is a self-adjoint nonnegative operator on a separable Hilbert space $H$,

- the coefficients $c_{n}:[0, T] \rightarrow \mathbb{R}$ satisfy the strict hyperbolicity assumption (1.7) with the same constants $\mu_{1}$ and $\mu_{2}$, and the $\omega$-continuity assumption (2.4) with the same continuity modulus $\omega(x)$, 
- $c_{n}(t)$ pointwise converges to $c_{\infty}(t)$,

- $\sigma \in[0,1 / 2]$ and $\delta>0$ are two real numbers satisfying (3.7),

Then $u_{n} \rightarrow u_{\infty}$ in the sense of (4.6).

We observe that in both cases the limit coefficient $c_{\infty}(t)$ satisfies the same assumptions of the approximating coefficients $c_{n}(t)$.

The rest of this section is devoted to the proof of these results. A careful inspection of the argument reveals that in both cases the pointwise convergence assumption (4.3) can be weakened to convergence in $L^{2}((0, T))$.

\section{Estimates on components}

In this section we provide estimates for solutions to the family of non-homogeneous linear ordinary differential equations

$$
w^{\prime \prime}(t)+2 \delta \lambda^{2 \sigma} w^{\prime}(t)+\lambda^{2} c(t) w(t)=f(t),
$$

with null initial data

$$
w(0)=w^{\prime}(0)=0 .
$$

Our interest is motivated by the fact that Fourier components of the difference $u_{n}(t)-u_{\infty}(t)$ are solutions to problems of this type.

Lemma 4.3 (Supercritical dissipation). Let $T>0$, and let $w(t)$ be the solution to problem (4.7)-(4.8) under the following assumptions:

- the coefficient $c:[0, T] \rightarrow \mathbb{R}$ is measurable and satisfies (1.11),

- $\sigma$ and $\delta$ are two positive real numbers satisfying the assumptions of Theorem 4.1,

- $\lambda \geq 0$ and $f \in L^{2}((0, T), \mathbb{R})$.

Then there exist two constants $\Gamma_{1}$ and $\Gamma_{2}$ such that

$$
\left|w^{\prime}(t)\right|^{2}+\left(1+\lambda^{2}\right)|w(t)|^{2} \leq \Gamma_{1} \exp \left(\Gamma_{2} t\right) \int_{0}^{t}|f(s)|^{2} d s \quad \forall t \in[0, T] .
$$

The constants $\Gamma_{1}$ and $\Gamma_{2}$ depend only on $\delta, \sigma$, and $\mu_{2}$ (in particular they are independent of $\lambda, f$, and $T$ ). 
Proof Let us consider the energy

$$
E(t):=\left|w^{\prime}(t)\right|^{2}+\left(1+2 \delta^{2} \lambda^{4 \sigma}\right)|w(t)|^{2}+2 \delta \lambda^{2 \sigma} w(t) w^{\prime}(t) .
$$

The constants $k_{1}, \ldots, k_{4}$ we introduce in the sequel are positive numbers depending only on $\delta, \sigma$, and $\mu_{2}$.

Since

$$
\left|2 \delta \lambda^{2 \sigma} w(t) w^{\prime}(t)\right| \leq \frac{3}{4}\left|w^{\prime}(t)\right|^{2}+\frac{4}{3} \delta^{2} \lambda^{4 \sigma}|w(t)|^{2},
$$

and since $\lambda^{2} \leq 1+\lambda^{4 \sigma}$ (because $\sigma \geq 1 / 2$ ), it turns out that

$$
\left|w^{\prime}(t)\right|^{2}+\left(1+\lambda^{4 \sigma}+\lambda^{2}\right)|w(t)|^{2} \leq k_{1} E(t) .
$$

The time-derivative of $E(t)$ is

$$
\begin{aligned}
E^{\prime}(t)= & -2\left(\delta \lambda^{2 \sigma}\left|w^{\prime}(t)\right|^{2}+\delta \lambda^{2 \sigma+2} c(t)|w(t)|^{2}+\lambda^{2} c(t) w(t) w^{\prime}(t)\right) \\
& +2 w^{\prime}(t) f(t)+2 w^{\prime}(t) w(t)+2 \delta \lambda^{2 \sigma} w(t) f(t) .
\end{aligned}
$$

Due to (4.11), the last three terms can be estimated as follows

$$
\begin{aligned}
2 w^{\prime}(t) f(t) & +2 w^{\prime}(t) w(t)+2 \delta \lambda^{2 \sigma} w(t) f(t) \\
& \leq 2\left|w^{\prime}(t)\right|^{2}+\left(1+\delta^{2} \lambda^{4 \sigma}\right)|w(t)|^{2}+2|f(t)|^{2} \\
& \leq k_{2} E(t)+2|f(t)|^{2} .
\end{aligned}
$$

We claim that

$$
-2\left(\delta \lambda^{2 \sigma}\left|w^{\prime}(t)\right|^{2}+\delta \lambda^{2 \sigma+2} c(t)|w(t)|^{2}+\lambda^{2} c(t) w(t) w^{\prime}(t)\right) \leq k_{3} E(t)
$$

Indeed, this inequality is equivalent to

$$
A\left|w^{\prime}(t)\right|^{2}+B|w(t)|^{2}+2 C w(t) w^{\prime}(t) \geq 0,
$$

where for the sake of shortness we set

$$
A:=k_{3}+2 \delta \lambda^{2 \sigma}, \quad B:=k_{3}+2 k_{3} \delta^{2} \lambda^{4 \sigma}+2 \delta \lambda^{2 \sigma+2} c(t), \quad C:=k_{3} \delta \lambda^{2 \sigma}+\lambda^{2} c(t) .
$$

The left-hand side of (4.15) is a quadratic form in $w^{\prime}(t)$ and $w(t)$. The coefficients $A$ and $B$ are positive. Therefore, this quadratic form is nonnegative for all values of $w^{\prime}(t)$ and $w(t)$ if and only if $A B \geq C^{2}$. With some algebra, this condition turns out to be equivalent to

$$
4 \delta^{2} \lambda^{4 \sigma+2} c(t)+k_{3}^{2}+4 k_{3} \delta^{3} \lambda^{6 \sigma}+2 k_{3} \delta \lambda^{2 \sigma}+k_{3}^{2} \delta^{2} \lambda^{4 \sigma} \geq \lambda^{4} c^{2}(t) .
$$

If $\sigma=1 / 2$ and $4 \delta^{2} \geq \mu_{2}$, then

$$
4 \delta^{2} \lambda^{4 \sigma+2} c(t)=4 \delta^{2} \lambda^{4} c(t) \geq \mu_{2} \lambda^{4} c(t) \geq \lambda^{4} c^{2}(t),
$$


and hence even the first term in the left-hand side of (4.16) is greater than or equal to the right-hand side, independently of $k_{3}$. The same is true if $\sigma>1 / 2$ and $4 \delta^{2} \lambda^{4 \sigma-2} \geq \mu_{2}$.

If $\sigma>1 / 2$ and $4 \delta^{2} \lambda^{4 \sigma-2} \leq \mu_{2}$, then $\lambda$ is bounded in terms of $\delta$ and $\mu_{2}$, and hence the term $k_{3}^{2}$ alone in the left-hand side of (4.16) is greater than or equal to the right-hand side, provided that $k_{3}$ is suitably chosen. This completes the proof of (4.14).

Plugging (4.13) and (4.14) into (4.12), we deduce that

$$
E^{\prime}(t) \leq k_{4} E(t)+2|f(t)|^{2}
$$

and hence

$$
E(t) \leq e^{k_{4} t}\left(E(0)+2 \int_{0}^{t} e^{-k_{4} s}|f(s)|^{2} d s\right) \quad \forall t \geq 0 .
$$

Since $E(0)=0$ because of (4.8), ignoring the exponential inside the integral we conclude that

$$
E(t) \leq 2 e^{k_{4} t} \int_{0}^{t}|f(s)|^{2} d s \quad \forall t \geq 0 .
$$

At this point (4.9), with $\Gamma_{1}:=2 k_{1}$ and $\Gamma_{2}:=k_{4}$, follows from (4.11).

Lemma 4.4 (Subcritical dissipation). Let $T>0$, and let $w(t)$ be the solution to problem (4.7)-(4.8) under the following assumptions:

- the coefficient $c:[0, T] \rightarrow \mathbb{R}$ satisfies the strict hyperbolicity assumption (1.7) and the $\omega$-continuity assumption (2.4),

- $\sigma$ and $\delta$ are two positive real numbers satisfying the assumptions of Theorem 4.2,

- $\lambda \geq 0$ and $f \in L^{2}((0, T), \mathbb{R})$.

Then there exist two constants $\Gamma_{3}$ and $\Gamma_{4}$ such that

$$
\left|w^{\prime}(t)\right|^{2}+\left(1+\lambda^{2}\right)|w(t)|^{2} \leq \Gamma_{3} \exp \left(\Gamma_{4} t\right) \int_{0}^{t}|f(s)|^{2} d s \quad \forall t \in[0, T] .
$$

The constants $\Gamma_{3}$ and $\Gamma_{4}$ depend only on $\delta, \sigma, \mu_{1}$, and on the continuity modulus $\omega(x)$ (in particular they are independent of $\lambda, f$, and $T$ ).

Proof Let us extend $c(t)$ beyond $T$ by setting $c(t):=c(T)$ for $t \geq T$. For every $\varepsilon>0$, let us introduce the regularized coefficient

$$
c_{\varepsilon}(t):=\frac{1}{\varepsilon} \int_{t}^{t+\varepsilon} c(s) d s \quad \forall t \in[0, T] .
$$

It is easy to see that $c_{\varepsilon} \in C^{1}([0, T], \mathbb{R})$ and satisfies the following estimates:

$$
\mu_{1} \leq c_{\varepsilon}(t) \leq \mu_{2} \quad \forall t \in[0, T]
$$




$$
\begin{gathered}
\left|c(t)-c_{\varepsilon}(t)\right| \leq \omega(\varepsilon) \quad \forall t \in[0, T], \\
\left|c_{\varepsilon}^{\prime}(t)\right| \leq \frac{\omega(\varepsilon)}{\varepsilon} \quad \forall t \in[0, T] .
\end{gathered}
$$

The constants $\nu$, and $k_{1}, \ldots, k_{4}$ we introduce in the sequel are positive numbers depending only on $\delta, \sigma, \mu_{1}$, and on the continuity modulus $\omega(x)$.

To begin with, we fix $\nu \geq 1$ such that (3.9) holds true. Such a value exists due to assumption (3.7). Then we set

$$
\varepsilon(\lambda):= \begin{cases}1 & \text { if } \lambda<\nu \\ 1 / \lambda & \text { if } \lambda \geq \nu\end{cases}
$$

and we consider the energy

$$
E_{\lambda}(t):=\left|w^{\prime}(t)\right|^{2}+\left(1+2 \delta^{2} \lambda^{4 \sigma}+\lambda^{2} c_{\varepsilon(\lambda)}(t)\right)|w(t)|^{2}+2 \delta \lambda^{2 \sigma} w(t) w^{\prime}(t) .
$$

The last term can be estimated as in (4.10). Therefore, keeping (4.17) into account, it follows that

$$
\left|w^{\prime}(t)\right|^{2}+\left(1+\lambda^{2}\right)|w(t)|^{2} \leq k_{1} E_{\lambda}(t) .
$$

The time-derivative of $E_{\lambda}(t)$ is

$$
\begin{aligned}
E_{\lambda}^{\prime}(t)= & -2\left(\delta \lambda^{2 \sigma}\left|w^{\prime}(t)\right|^{2}+\delta \lambda^{2 \sigma+2} c(t)|w(t)|^{2}+\lambda^{2} c(t) w(t) w^{\prime}(t)\right) \\
& +\lambda^{2} c_{\varepsilon(\lambda)}^{\prime}(t)|w(t)|^{2}+2 \lambda^{2} c_{\varepsilon(\lambda)}(t) w(t) w^{\prime}(t) \\
& +2 w^{\prime}(t) f(t)+2 w^{\prime}(t) w(t)+2 \delta \lambda^{2 \sigma} w(t) f(t) .
\end{aligned}
$$

Let $L_{1}, L_{2}, L_{3}$ denote the three lines in the expression of $E_{\lambda}^{\prime}(t)$. To begin with, we observe that

$$
L_{3} \leq 2\left|w^{\prime}(t)\right|^{2}+\left(1+\delta^{2} \lambda^{4 \sigma}\right)|w(t)|^{2}+2|f(t)|^{2} .
$$

Since $\lambda^{4 \sigma} \leq 1+\lambda^{2}$ (because $\sigma \leq 1 / 2$ ), from (4.20) we deduce that

$$
L_{3} \leq k_{2} E(t)+2|f(t)|^{2} \quad \forall t \in[0, T] .
$$

Now we claim that

$$
L_{1}+L_{2} \leq k_{3} E(t) \quad \forall t \in[0, T] .
$$

Indeed, this inequality is equivalent to

$$
A\left|w^{\prime}(t)\right|^{2}+B|w(t)|^{2}+2 C w(t) w^{\prime}(t) \geq 0,
$$

where for the sake of shortness we set

$$
\begin{gathered}
A:=k_{3}+2 \delta \lambda^{2 \sigma}, \quad C:=k_{3} \delta \lambda^{2 \sigma}+\lambda^{2}\left(c(t)-c_{\varepsilon(\lambda)}(t)\right), \\
B:=2 k_{3} \delta^{2} \lambda^{4 \sigma}+k_{3}+2 \delta \lambda^{2 \sigma+2} c(t)+k_{3} \lambda^{2} c_{\varepsilon(\lambda)}(t)-\lambda^{2} c_{\varepsilon(\lambda)}^{\prime}(t) .
\end{gathered}
$$


The left-hand side of (4.24) is a quadratic form in $w^{\prime}(t)$ and $w(t)$. The coefficient $A$ is positive. Therefore, this quadratic form is nonnegative for all values of $w^{\prime}(t)$ and $w(t)$ if and only if $A B \geq C^{2}$. With some algebra, this condition turns out to be equivalent to

$$
\begin{array}{rl}
4 \delta^{2} \lambda^{4 \sigma+2} & c(t)+4 k_{3} \delta \lambda^{2 \sigma+2} c_{\varepsilon(\lambda)}(t)+k_{3}^{2}+4 k_{3} \delta^{3} \lambda^{6 \sigma}+2 k_{3} \delta \lambda^{2 \sigma}+k_{3}^{2} \delta^{2} \lambda^{4 \sigma}+ \\
& +k_{3}^{2} \lambda^{2} c_{\varepsilon(\lambda)}(t) \geq \lambda^{4}\left(c(t)-c_{\varepsilon(\lambda)}(t)\right)^{2}+2 \delta \lambda^{2 \sigma+2} c_{\varepsilon(\lambda)}^{\prime}(t)+k_{3} \lambda^{2} c_{\varepsilon(\lambda)}^{\prime}(t) .
\end{array}
$$

When $\lambda \geq \nu$ we chose $\varepsilon(\lambda)=1 / \lambda$, so that (4.18) and (4.19) read as

$$
\left|c(t)-c_{\varepsilon(\lambda)}(t)\right| \leq \omega\left(\frac{1}{\lambda}\right), \quad\left|c_{\varepsilon(\lambda)}^{\prime}(t)\right| \leq \lambda \omega\left(\frac{1}{\lambda}\right) .
$$

Therefore, since (3.9) holds true for $\lambda \geq \nu$, it follows that

$$
\begin{gathered}
4 \delta^{2} \lambda^{4 \sigma+2} c(t) \geq 4 \delta^{2} \mu_{1} \lambda^{4 \sigma+2} \geq \lambda^{4} \omega^{2}\left(\frac{1}{\lambda}\right)+2 \delta \lambda^{2 \sigma+3} \omega\left(\frac{1}{\lambda}\right) \\
\geq \lambda^{4}\left(c(t)-c_{\varepsilon(\lambda)}(t)\right)^{2}+2 \delta \lambda^{2 \sigma+2} c_{\varepsilon(\lambda)}^{\prime}(t),
\end{gathered}
$$

and

$$
4 k_{3} \delta \lambda^{2 \sigma+2} c_{\varepsilon(\lambda)}(t) \geq 4 \delta^{2} \mu_{1} \cdot \frac{k_{3} \lambda^{2 \sigma+2}}{\delta} \geq 2 \delta \lambda^{1-2 \sigma} \omega\left(\frac{1}{\lambda}\right) \cdot \frac{k_{3} \lambda^{2 \sigma+2}}{\delta} \geq k_{3} \lambda^{2} c_{\varepsilon(\lambda)}^{\prime}(t) .
$$

As a consequence, when $\lambda \geq \nu$ the sum of the first two terms in the left-hand side of (4.25) is greater than or equal to the whole right-hand side, independently of $k_{3}$.

When $\lambda<\nu$ we chose $\varepsilon(\lambda)=1$. Thanks to (4.18) and (4.19) with $\varepsilon=1$, the right-hand side of (4.25) is less than or equal to

$$
\nu^{4} \omega^{2}(1)+2 \delta \nu^{2 \sigma+2} \omega(1)+k_{3} \nu^{2} \omega(1) .
$$

As a consequence, the third term in the left-hand side of (4.25), namely $k_{3}^{2}$, is greater than or equal to the whole right-hand side, provided that $k_{3}$ is large enough. This completes the proof of (4.23).

Plugging (4.22) and (4.23) into (4.21), we deduce that

$$
E_{\lambda}^{\prime}(t) \leq k_{4} E_{\lambda}(t)+2|f(t)|^{2} \quad \forall t \in[0, T] .
$$

The conclusion follows exactly as in the proof of Lemma 4.3

\section{Proof of Theorem 4.1}

The result is established in two steps. In the first one, we prove the conclusion under the more restrictive assumption that $\left(u_{0}, u_{1}\right) \in D(A) \times D\left(A^{1 / 2}\right)$. In this case a stronger result holds true, in the sense that the norm of $u_{n}-u_{\infty}$ in the energy space can be estimated in terms of the norm of $c_{n}-c_{\infty}$ in $L^{2}((0, T), \mathbb{R})$. In the second step we apply an approximation procedure in order to obtain the conclusion for all initial data, abandoning the possibility to estimate the convergence rate. 
Convergence for more regular data Let us assume that $\left(u_{0}, u_{1}\right) \in D(A) \times D\left(A^{1 / 2}\right)$. We prove that there exists a constant $\Gamma$, depending only on $\delta, \sigma, \mu_{2}$ and $T$, such that

$$
\begin{aligned}
\mid u_{\infty}^{\prime}(t) & -\left.u_{n}^{\prime}(t)\right|^{2}+\left|A^{1 / 2}\left(u_{\infty}(t)-u_{n}(t)\right)\right|^{2} \\
& \leq \Gamma\left(\left|A^{1 / 2} u_{1}\right|^{2}+\left|A u_{0}\right|^{2}\right) \int_{0}^{T}\left|c_{n}(s)-c_{\infty}(s)\right|^{2} d s
\end{aligned}
$$

for every $t \in[0, T]$.

To this end, we introduce the difference $w_{n}(t):=u_{\infty}(t)-u_{n}(t)$, which turns out to be a solution to

$$
w_{n}^{\prime \prime}(t)+2 \delta A^{\sigma} w_{n}^{\prime}(t)+c_{\infty}(t) A w_{n}(t)=\left(c_{n}(t)-c_{\infty}(t)\right) A u_{n}(t),
$$

with null initial data $w_{n}(0)=w_{n}^{\prime}(0)=0$. We also consider the components $w_{n, k}(t)$ of $w_{n}(t)$ with respect to the orthonormal system $\left\{e_{k}\right\}$. If $u_{n, k}(t)$ are the corresponding components of $u_{n}(t)$, it turns out that $w_{n, k}(t)$ is the solution to the ordinary differential equation

$$
w_{n, k}^{\prime \prime}(t)+2 \delta \lambda_{k}^{2 \sigma} w_{n, k}^{\prime}(t)+\lambda_{k}^{2} c_{\infty}(t) w_{n, k}(t)=\lambda_{k}^{2}\left(c_{n}(t)-c_{\infty}(t)\right) u_{n, k}(t),
$$

with null initial data $w_{n, k}(0)=w_{n, k}^{\prime}(0)=0$. Therefore, we can apply Lemma 4.3 with

$$
w(t):=w_{n, k}(t), \quad \lambda:=\lambda_{k}, \quad c(t):=c_{\infty}(t), \quad f(t):=\lambda_{k}^{2}\left(c_{n}(t)-c_{\infty}(t)\right) u_{n, k}(t) .
$$

We obtain that

$$
\left|w_{n, k}^{\prime}(t)\right|^{2}+\lambda_{k}^{2}\left|w_{n, k}(t)\right|^{2} \leq \Gamma_{1} \exp \left(\Gamma_{2} t\right) \int_{0}^{t} \lambda_{k}^{4}\left|c_{n}(s)-c_{\infty}(s)\right|^{2} \cdot\left|u_{n, k}(s)\right|^{2} d s,
$$

where $\Gamma_{1}$ and $\Gamma_{2}$ do not depend on $k$. Therefore, summing over all $k$ 's we deduce that

$$
\left|w_{n}^{\prime}(t)\right|^{2}+\left|A^{1 / 2} w_{n}(t)\right|^{2} \leq \Gamma_{1} \exp \left(\Gamma_{2} t\right) \int_{0}^{t}\left|c_{n}(s)-c_{\infty}(s)\right|^{2} \cdot\left|A u_{n}(s)\right|^{2} d s .
$$

In order to estimate $\left|A u_{n}(s)\right|$, we choose $\nu \geq 1$ satisfying (3.3) (we point out that $\nu$ depends only on $\delta, \sigma$ and $\left.\mu_{2}\right)$, and we apply Theorem A to the function $A^{1 / 2} u_{n}(t)$, which is again a solution to equation (4.1) thanks to linearity. We obtain that

$$
\left|A u_{n}(t)\right|^{2} \leq K_{1}\left(\delta, \mu_{2}\right) e^{\nu\left(1+\mu_{2}\right) T}\left(\left|A^{1 / 2} u_{1}\right|^{2}+\left|A u_{0}\right|^{2}\right) \quad \forall t \in[0, T] .
$$

Plugging this estimate into (4.27), we finally deduce (4.26). 
Convergence for general data Let us consider now an initial condition $\left(u_{0}, u_{1}\right) \in$ $D\left(A^{1 / 2}\right) \times H$. We show that, for every $\eta>0$, there exists $n_{0} \in \mathbb{N}$ such that

$$
\left|u_{n}^{\prime}(t)-u_{\infty}^{\prime}(t)\right|^{2}+\left|A^{1 / 2}\left(u_{n}(t)-u_{\infty}(t)\right)\right|^{2} \leq \eta \quad \forall t \in[0, T], \quad \forall n \geq n_{0} .
$$

To this end, we exploit a classical approximation argument. We choose $\nu \geq 1$ satisfying (3.3), and then we choose $\left(v_{0}, v_{1}\right) \in D(A) \times D\left(A^{1 / 2}\right)$ such that

$$
\left|u_{1}-v_{1}\right|^{2}+\left|A^{1 / 2}\left(u_{0}-v_{0}\right)\right|^{2} \leq \frac{\eta}{9 K_{1}\left(\delta, \mu_{2}\right) e^{\nu\left(1+\mu_{2}\right) T}}
$$

where $K_{1}\left(\delta, \mu_{2}\right)$ is again the constant of Theorem A.

Let $v_{n}(t)$ and $v_{\infty}(t)$ denote the solutions to (4.1) and (4.4), respectively, with initial data $v_{n}(0)=v_{\infty}(0)=v_{0}$ and $v_{n}^{\prime}(0)=v_{\infty}^{\prime}(0)=v_{1}$. Since $u_{n}(t)-v_{n}(t)$ is again a solution to (4.1), from (3.4) and (4.29) it follows that

$$
\left|u_{n}^{\prime}(t)-v_{n}^{\prime}(t)\right|^{2}+\left|A^{1 / 2}\left(u_{n}(t)-v_{n}(t)\right)\right|^{2} \leq \frac{\eta}{9} \quad \forall t \in[0, T] .
$$

Since $u_{\infty}(t)-v_{\infty}(t)$ is again a solution to (4.4), from (3.4) and (4.29) it follows that

$$
\left|u_{\infty}^{\prime}(t)-v_{\infty}^{\prime}(t)\right|^{2}+\left|A^{1 / 2}\left(u_{\infty}(t)-v_{\infty}(t)\right)\right|^{2} \leq \frac{\eta}{9} \quad \forall t \in[0, T] .
$$

Finally, since $\left(v_{0}, v_{1}\right) \in D(A) \times D\left(A^{1 / 2}\right)$, we can apply (4.26) to $v_{n}(t)$ and $v_{\infty}(t)$. It follows that there exists $n_{0} \in \mathbb{N}$ such that

$$
\left|v_{n}^{\prime}(t)-v_{\infty}^{\prime}(t)\right|^{2}+\left|A^{1 / 2}\left(v_{n}(t)-v_{\infty}(t)\right)\right|^{2} \leq \frac{\eta}{9} \quad \forall t \in[0, T] \quad \forall n \geq n_{0} .
$$

Since

$$
\begin{aligned}
\left|u_{n}^{\prime}(t)-u_{\infty}^{\prime}(t)\right|^{2}+ & \left|A^{1 / 2}\left(u_{n}(t)-u_{\infty}(t)\right)\right|^{2} \\
\leq \quad 3 & \left(\left|u_{n}^{\prime}(t)-v_{n}^{\prime}(t)\right|^{2}+\left|A^{1 / 2}\left(u_{n}(t)-v_{n}(t)\right)\right|^{2}\right. \\
& +\left|v_{n}^{\prime}(t)-v_{\infty}^{\prime}(t)\right|^{2}+\left|A^{1 / 2}\left(v_{n}(t)-v_{\infty}(t)\right)\right|^{2} \\
& \left.+\left|u_{\infty}^{\prime}(t)-v_{\infty}^{\prime}(t)\right|^{2}+\left|A^{1 / 2}\left(u_{\infty}(t)-v_{\infty}(t)\right)\right|^{2}\right),
\end{aligned}
$$

conclusion (4.28) follows from (4.30), (4.31), and (4.32). This proves (4.6).

\section{Proof of Theorem 4.2}

Same proof of Theorem 4.1, the only difference being that the key estimates come from Theorem B and Lemma 4.4, instead of Theorem A and Lemma 4.3, and the constant $\Gamma$ in (4.26) now depends on $\delta, \sigma, \mu_{1}, \mu_{2}, T$, and on the continuity modulus $\omega$. 


\section{$5 \quad$ Interpolation spaces}

In this section we introduce a family of interpolation spaces between the energy space $D\left(A^{1 / 2}\right) \times H$ and the standard space for Kirchhoff equations $D\left(A^{3 / 4}\right) \times D\left(A^{1 / 4}\right)$. We prove three results. First of all, there is propagation of regularity for problem (1.10)(1.2), in the sense that the problem is well-posed in these intermediate spaces whenever it is well-posed in the energy space. More important, the continuity modulus of the function $t \rightarrow\left|A^{1 / 2} u(t)\right|^{2}$ depends only on the regularity of initial data in these interpolation spaces, and not on the regularity of $c(t)$. Finally, we show that any pair of initial conditions $\left(u_{0}, u_{1}\right)$ in the energy space lies actually in a suitable interpolation space.

Roughly speaking, the role of these interpolation spaces between the energy space and $D\left(A^{3 / 4}\right) \times D\left(A^{1 / 4}\right)$ is the same as the role of functions with a given continuity modulus between the space of all continuous functions and the space of Lipschitz continuous functions. The definition itself relies on the notion of continuity modulus.

Definition 5.1. Let $\omega(x)$ be a continuity modulus. For every $\left(u_{0}, u_{1}\right) \in D\left(A^{1 / 2}\right) \times H$ we set

$$
\left\|\left(u_{0}, u_{1}\right)\right\|_{\omega}^{2}:=\sum_{k \in K_{0}} \frac{1}{\omega\left(1 / \lambda_{k}\right)}\left(\left|u_{1 k}\right|^{2}+\lambda_{k}^{2}\left|u_{0 k}\right|^{2}\right),
$$

where $K_{0}$ denotes the set of nonnegative integers $k$ such that $\lambda_{k}>0$, and $u_{0 k}$ and $u_{1 k}$ denote the components of $u_{0}$ and $u_{1}$ with respect to the usual orthonormal system. Then we define the space

$$
V_{\omega}:=\left\{\left(u_{0}, u_{1}\right) \in D\left(A^{1 / 2}\right) \times H:\left\|\left(u_{0}, u_{1}\right)\right\|_{\omega}^{2}<+\infty\right\} .
$$

It is easy to see that

$$
D\left(A^{3 / 4}\right) \times D\left(A^{1 / 4}\right) \subseteq V_{\omega} \subseteq D\left(A^{1 / 2}\right) \times H .
$$

Moreover, $V_{\omega}$ is actually a vector space, $\left\|\left(u_{0}, u_{1}\right)\right\|_{\omega}$ is a seminorm, and the full norm

$$
\left(\left|u_{1}\right|^{2}+\left|u_{0}\right|^{2}+\left\|\left(u_{0}, u_{1}\right)\right\|_{\omega}^{2}\right)^{1 / 2}
$$

induces a Hilbert space structure on $V_{\omega}$.

When $\omega(x)=x^{4 \alpha}$ for some $\alpha \in[0,1 / 4]$, the space $V_{\omega}$ is just $D\left(A^{\alpha+1 / 2}\right) \times D\left(A^{\alpha}\right)$.

Since in this paper we are dealing with continuity moduli in several different contexts, from now on we write $\omega_{d}(x)$ to denote continuity moduli involved in interpolation spaces (here " $d$ " stands for "data").

\subsection{Propagation of regularity}

When problem (1.10)-(1.2) generates a continuous semigroup in the energy space, then every pair of initial conditions in $V_{\omega_{d}}$ gives rise to a solution lying in the same space for all positive times.

Let us start with the case $\sigma \geq 1 / 2$. 
Proposition 5.2 (Supercritical case - Regularity in interpolation spaces). Let $T>0$, and let $u(t)$ be the solution in $[0, T]$ to problem (1.10)-(1.2) under the same assumption of Theorem A.

Let us assume in addition that $\left(u_{0}, u_{1}\right) \in V_{\omega_{d}}$ for some continuity modulus $\omega_{d}(x)$.

Then $\left(u, u^{\prime}\right) \in C^{0}\left([0, T], V_{\omega_{d}}\right)$ and for every $\nu \geq 1$ satisfying (3.3) it turns out that

$$
\left\|\left(u(t), u^{\prime}(t)\right)\right\|_{\omega_{d}}^{2} \leq K_{1}\left(\delta, \mu_{2}\right) e^{\nu\left(1+\mu_{2}\right) t}\left\|\left(u_{0}, u_{1}\right)\right\|_{\omega_{d}}^{2} \quad \forall t \in[0, T],
$$

where $K_{1}\left(\delta, \mu_{2}\right)$ is the constant of Theorem $A$.

Proof Let $\left\{u_{k}(t)\right\}$ denote the components of $u(t)$ with respect to the usual orthonormal system $\left\{e_{k}\right\}$, and let $\left\{u_{0 k}\right\}$ and $\left\{u_{1 k}\right\}$ denote the corresponding components of initial conditions. Since we are in the assumptions of Theorem A, we can estimate these components as in (3.12) and (3.13). Since $K_{1}\left(\delta, \mu_{2}\right) \geq 1$, we can combine these two estimates and deduce that

$$
\left|u_{k}^{\prime}(t)\right|^{2}+\lambda_{k}^{2}\left|u_{k}(t)\right|^{2} \leq K_{1}\left(\delta, \mu_{2}\right) e^{\nu\left(1+\mu_{2}\right) t}\left(\left|u_{1 k}\right|^{2}+\lambda_{k}^{2}\left|u_{0 k}\right|^{2}\right) \quad \forall t \in[0, T],
$$

independently of $k$. Dividing by $\omega\left(1 / \lambda_{k}\right)$, and summing over $K_{0}$ (the set of indices $k$ with $\lambda_{k} \neq 0$ ), we obtain (5.1).

The same estimate shows also the uniform convergence of the series

$$
\sum_{k \in K_{0}} \frac{\lambda_{k}^{2}}{\omega_{d}\left(1 / \lambda_{k}\right)}\left|u_{k}(t)\right|^{2}, \quad \sum_{k \in K_{0}} \frac{1}{\omega_{d}\left(1 / \lambda_{k}\right)}\left|u_{k}^{\prime}(t)\right|^{2},
$$

which proves the continuity of the pair $\left(u, u^{\prime}\right)$ with values in $V_{\omega_{d}}$.

The result for the subcritical case is analogous. Here we state explicitly a time independent estimate for high-frequency components in the same spirit of (3.11). This estimate is crucial in the proof of the global existence statement of Theorem 2.2. An analogous estimate holds true also in the supercritical case, but we do not need it in the sequel.

Proposition 5.3 (Subcritical case - Regularity in interpolation spaces). Let $T>0$, and let $u(t)$ be the solution in $[0, T]$ to problem (1.10)-(1.2) under the same assumption of Theorem $B$.

Let us assume in addition that $\left(u_{0}, u_{1}\right) \in V_{\omega_{d}}$ for some continuity modulus $\omega_{d}(x)$.

Then $\left(u, u^{\prime}\right) \in C^{0}\left([0, T], V_{\omega_{d}}\right)$ and for every $\nu \geq 1$ satisfying (3.9) it turns out that

$$
\left\|\left(u(t), u^{\prime}(t)\right)\right\|_{\omega_{d}}^{2} \leq K_{2}\left(\delta, \mu_{1}, \mu_{2}\right) e^{\nu\left(1+\mu_{2}\right) t}\left\|\left(u_{0}, u_{1}\right)\right\|_{\omega_{d}}^{2} \quad \forall t \in[0, T],
$$

where $K_{2}\left(\delta, \mu_{1}, \mu_{2}\right)$ is the constant of Theorem $B$.

Moreover, the high-frequency component of $u(t)$ satisfies

$$
\left\|\left(u_{\nu,+}(t), u_{\nu,+}^{\prime}(t)\right)\right\|_{\omega_{d}}^{2} \leq K_{2}\left(\delta, \mu_{1}, \mu_{2}\right)\left\|\left(u_{0, \nu,+}, u_{1, \nu,+}\right)\right\|_{\omega_{d}}^{2} \quad \forall t \in[0, T]
$$


Proof Same proof of Proposition 5.2, this time starting from estimates (3.12) and (3.14), which hold true under the assumptions of Theorem B.

Remark 5.4. From (2.1) it follows that $\omega_{d}\left(1 / \lambda_{k}\right) \geq \omega_{d}(1 / \nu)$ whenever $\lambda_{k} \leq \nu$. Therefore, both in the supercritical and in the subcritical case, the low-frequency components can be estimated in terms of the usual energy as follows

$$
\left\|\left(u_{\nu,-}(t), u_{\nu,-}^{\prime}(t)\right)\right\|_{\omega_{d}}^{2} \leq \frac{1}{\omega_{d}(1 / \nu)}\left(\left|u_{\nu,-}^{\prime}(t)\right|^{2}+\left|A^{1 / 2} u_{\nu,-}(t)\right|^{2}\right) \quad \forall t \in[0, T] .
$$

Also this estimate is crucial in the proof of the global existence statement of Theorem 2.2.

\subsection{Interpolation spaces vs time regularity}

It is well-known that space regularity and time regularity of solutions to hyperbolic problems are strongly related. The following result clarifies the connection between the interpolation space $V_{\omega_{d}}$ and the continuity modulus of the function $t \rightarrow\left|A^{1 / 2} u(t)\right|^{2}$.

Proposition 5.5 (Continuity modulus of $\left.\left|A^{1 / 2} u(t)\right|^{2}\right)$. Let $\omega_{d}(x)$ be a continuity modulus, let $T>0$, and let $u:[0, T] \rightarrow H$ be a function with the regularity (3.2).

Let us assume that $\left(u(t), u^{\prime}(t)\right) \in V_{\omega_{d}}$ for every $t \in[0, T]$, and there exists a constant $L$ such that

$$
\left\|\left(u(t), u^{\prime}(t)\right)\right\|_{\omega_{d}}^{2} \leq L \quad \forall t \in[0, T] .
$$

Then it turns out that

$$
\left.|| A^{1 / 2} u(a)\right|^{2}-\left|A^{1 / 2} u(b)\right|^{2} \mid \leq 3 L \omega_{d}(|a-b|) \quad \forall(a, b) \in[0, T]^{2} .
$$

Proof Let $u_{k}(t)$ denote the components of $u(t)$ with respect to the usual orthonormal system. For every $\varepsilon>0$, let $K_{\varepsilon}^{-}$denote the set of nonnegative integers $k$ such that $0<\lambda_{k} \leq 1 / \varepsilon$, and let $K_{\varepsilon}^{+}$denote the set of nonnegative integers $k$ such that $\lambda_{k}>1 / \varepsilon$. Let us set for simplicity

$$
g(t):=\left|A^{1 / 2} u(t)\right|^{2}=\sum_{k=0}^{\infty} \lambda_{k}^{2}\left|u_{k}(t)\right|^{2}, \quad g_{\varepsilon}(t):=\sum_{k \in K_{\varepsilon}^{-}} \lambda_{k}^{2}\left|u_{k}(t)\right|^{2} .
$$

The time-derivative of $g_{\varepsilon}(t)$ exists and is given by

$$
g_{\varepsilon}^{\prime}(t)=2 \sum_{k \in K_{\varepsilon}^{-}} \lambda_{k}^{2} u_{k}(t) u_{k}^{\prime}(t)
$$

and hence

$$
\left|g_{\varepsilon}^{\prime}(t)\right| \leq \sum_{k \in K_{\varepsilon}^{-}} \lambda_{k}\left(\left|u_{k}^{\prime}(t)\right|^{2}+\lambda_{k}^{2}\left|u_{k}(t)\right|^{2}\right)
$$


For every $k \in K_{\varepsilon}^{-}$it turns out that $1 / \lambda_{k} \geq \varepsilon$, and hence $\lambda_{k} \omega_{d}\left(1 / \lambda_{k}\right) \leq \omega_{d}(\varepsilon) / \varepsilon$ because of the monotonicity property (2.2) of the continuity modulus. Therefore, from (5.7) and assumption (5.5) we deduce that

$$
\left|g_{\varepsilon}^{\prime}(t)\right| \leq \frac{\omega_{d}(\varepsilon)}{\varepsilon} \sum_{k \in K_{\varepsilon}^{-}} \frac{1}{\omega_{d}\left(1 / \lambda_{k}\right)}\left(\left|u_{k}^{\prime}(t)\right|^{2}+\lambda_{k}^{2}\left|u_{k}(t)\right|^{2}\right) \leq L \frac{\omega_{d}(\varepsilon)}{\varepsilon}
$$

for every $t \in[0, T]$. In particular, from the mean value theorem it follows that

$$
\left|g_{\varepsilon}(a)-g_{\varepsilon}(b)\right| \leq|a-b| \cdot \max _{t \in[0, T]}\left|g_{\varepsilon}^{\prime}(t)\right| \leq L \frac{\omega_{d}(\varepsilon)}{\varepsilon} \cdot|a-b|
$$

for every $a$ and $b$ in $[0, T]$.

On the contrary, for every $k \in K_{\varepsilon}^{+}$it turns out that $1 / \lambda_{k}<\varepsilon$, and hence $\omega_{d}\left(1 / \lambda_{k}\right) \leq$ $\omega_{d}(\varepsilon)$ because of the monotonicity property (2.1) of the continuity modulus. It follows that

$$
\begin{aligned}
& \left|g(t)-g_{\varepsilon}(t)\right|=\sum_{k \in K_{\varepsilon}^{+}} \lambda_{k}^{2}\left|u_{k}(t)\right|^{2} \leq \sum_{k \in K_{\varepsilon}^{+}}\left(\left|u_{k}^{\prime}(t)\right|^{2}+\lambda_{k}^{2}\left|u_{k}(t)\right|^{2}\right) \\
& \leq \omega_{d}(\varepsilon) \sum_{k \in K_{\varepsilon}^{+}} \frac{1}{\omega_{d}\left(1 / \lambda_{k}\right)}\left(\left|u_{k}^{\prime}(t)\right|^{2}+\lambda_{k}^{2}\left|u_{k}(t)\right|^{2}\right) \leq L \omega_{d}(\varepsilon)
\end{aligned}
$$

for every $t \in[0, T]$. From this estimate and (5.8) we deduce that

$$
\begin{aligned}
|g(a)-g(b)| & \leq\left|g(a)-g_{\varepsilon}(a)\right|+\left|g_{\varepsilon}(a)-g_{\varepsilon}(b)\right|+\left|g_{\varepsilon}(b)-g(b)\right| \\
& \leq L \omega_{d}(\varepsilon)+L \frac{\omega_{d}(\varepsilon)}{\varepsilon} \cdot|a-b|+L \omega_{d}(\varepsilon)
\end{aligned}
$$

for every $a$ and $b$ in $[0, T]$, and every $\varepsilon>0$. Now it is enough to choose $\varepsilon:=|a-b|$, and (5.6) is proved.

\subsection{An intermediate space for each initial condition}

In the next result we show that every pair $\left(u_{0}, u_{1}\right)$ which is in $D\left(A^{\alpha+1 / 2}\right) \times D\left(A^{\alpha}\right)$ for some $\alpha \in[0,1 / 4)$ lies actually in a "better" space, namely a space $V_{\omega_{d}}$ corresponding to a continuity modulus $\omega_{d}(x)$ which tends to 0 faster than $x^{4 \alpha}$. It is a refinement of the classical calculus result according to which the terms of a converging series can always be multiplied by a diverging sequence obtaining again a converging series. We can also control the (semi)norm in the interpolation space in terms of the (semi)norm in the original space.

Proposition 5.6 (Customized interpolation spaces). For every $\alpha \in[0,1 / 4)$ and every $\left(u_{0}, u_{1}\right) \in D\left(A^{\alpha+1 / 2}\right) \times D\left(A^{\alpha}\right)$, there exists a continuity modulus $\omega_{d}(x)$ such that $\left(u_{0}, u_{1}\right) \in V_{\omega_{d}}$ and

$$
\omega_{d}(1)=1
$$




$$
\begin{gathered}
\omega_{d}(x) \leq x^{4 \alpha} \quad \forall x \geq 0, \\
\lim _{x \rightarrow 0^{+}} \frac{\omega_{d}(x)}{x^{4 \alpha}}=0 \\
\left\|\left(u_{0}, u_{1}\right)\right\|_{\omega_{d}}^{2} \leq 2\left(\left|A^{\alpha} u_{1}\right|^{2}+\left|A^{\alpha+1 / 2} u_{0}\right|^{2}\right) .
\end{gathered}
$$

Proof Let $u_{0 k}$ and $u_{1 k}$ denote the components of $u_{0}$ and $u_{1}$ with respect to the usual orthonormal system. Let us set for simplicity

$$
E_{k}:=\lambda_{k}^{4 \alpha}\left(\left|u_{1 k}\right|^{2}+\lambda_{k}^{2}\left|u_{0 k}\right|^{2}\right)
$$

and

$$
E:=\left|A^{\alpha} u_{1}\right|^{2}+\left|A^{\alpha+1 / 2} u_{0}\right|^{2}=\sum_{k=0}^{\infty} E_{k} .
$$

For every $n \in \mathbb{N}$, let us set

$$
A_{n}:=\left\{k \in \mathbb{N}: n \leq \lambda_{k}<n+1\right\}, \quad a_{n}:=\sum_{k \in A_{n}} E_{k} .
$$

Since

$$
\sum_{n=0}^{\infty} a_{n}=\sum_{k=0}^{\infty} E_{k}=E,
$$

there exists an increasing sequence $n_{h}$ of nonnegative integers such that $n_{0}=0$ and

$$
\sum_{n \geq n_{h}} a_{n} \leq \frac{E}{4^{h}} \quad \forall h \in \mathbb{N} .
$$

For every $h \in \mathbb{N}$, let us set

$$
B_{h}:=\left\{k \in \mathbb{N}: n_{h} \leq \lambda_{k}<n_{h+1}\right\} .
$$

Since $B_{h} \subseteq \cup_{n \geq n_{h}} A_{n}$, from (5.13) it turns out that

$$
\sum_{k \in B_{h}} E_{k} \leq \sum_{n \geq n_{h}} a_{n} \leq \frac{E}{4^{h}} \quad \forall h \in \mathbb{N} .
$$

Let us consider the sequence $\varphi_{h}$ defined by $\varphi_{0}=1, \varphi_{1}=1$, and

$$
\varphi_{h+1}=\min \left\{2^{h}, \frac{n_{h+1}}{n_{h}} \varphi_{h}\right\} \quad \forall h \geq 1 .
$$

From this definition it follows that

$$
\varphi_{h+1} \leq 2^{h}, \quad \varphi_{h+1} \geq \varphi_{h}, \quad \frac{n_{h+1}}{\varphi_{h+1}} \geq \frac{n_{h}}{\varphi_{h}}
$$


for every $h \in \mathbb{N}$. Moreover it turns out that

$$
\lim _{h \rightarrow+\infty} \varphi_{h}=+\infty
$$

Indeed, (5.16) is obvious if $\varphi_{h+1}=2^{h}$ for infinitely many indices. If not, it means that $\varphi_{h+1}=\varphi_{h} n_{h+1} / n_{h}$ for every $h$ greater than or equal to some $h_{0} \geq 1$. In this case an easy induction gives that

$$
\varphi_{h}=\frac{n_{h}}{n_{h_{0}}} \varphi_{h_{0}} \quad \forall h \geq h_{0}
$$

so that (5.16) follows from the fact that $n_{h} \rightarrow+\infty$.

Let us consider now the piecewise affine function $\varphi:[0,+\infty) \rightarrow \mathbb{R}$ such that $\varphi\left(n_{h}\right)=$ $\varphi_{h}$ for every $h \in \mathbb{N}$, namely the function defined by

$$
\varphi(x):=\varphi_{h}+\frac{\varphi_{h+1}-\varphi_{h}}{n_{h+1}-n_{h}}\left(x-n_{h}\right) \quad \text { if } n_{h} \leq x \leq n_{h+1} \text { for some } h \in \mathbb{N} .
$$

From the first relation in (5.15) it follows that

$$
\varphi(x) \leq 2^{h} \quad \forall x \in\left[n_{h}, n_{h+1}\right] .
$$

Moreover $\varphi(1)=1$ and

$$
\varphi(x) \geq 1 \quad \forall x \geq 0 .
$$

From the second relation in (5.15) it follows that $\varphi(x)$ is nondecreasing, and from (5.16) it follows that

$$
\lim _{x \rightarrow+\infty} \varphi(x)=+\infty
$$

Finally, from the third relation in (5.15) one can prove that

$$
\text { the function } x \rightarrow \frac{x}{\varphi(x)} \text { is nondecreasing }
$$

(it is enough to show that its derivative is nonnegative in each interval $\left(n_{h}, n_{h+1}\right)$ ).

Let us finally set

$$
\omega_{d}(x):= \begin{cases}0 & \text { if } x=0 \\ \frac{x^{4 \alpha}}{[\varphi(1 / x)]^{1-4 \alpha}} & \text { if } x>0\end{cases}
$$

We claim that $\omega_{d}(x)$ is a continuity modulus, and that (5.9) through (5.12) hold true. Equality (5.9) follows from the fact that $\varphi(1)=1$, while estimate (5.10) follows from (5.18). Since $4 \alpha<1$, from (5.19) we deduce that $\omega_{d}(x) \rightarrow 0$ as $x \rightarrow 0^{+}$, which proves that $\omega_{d}$ is continuous also in $x=0$, the only point in which continuity was nontrivial. The limit (5.11) follows from (5.19) because $4 \alpha<1$. The monotonicity property (2.1) of 
$\omega_{d}(x)$ follows from the fact that $\varphi(x)$ is nondecreasing, while the monotonicity property (2.2) is equivalent to (5.20) after the variable change $y:=1 / x$.

It remains to prove (5.12). To this end, we begin by observing that

$$
\begin{aligned}
\left\|\left(u_{0}, u_{1}\right)\right\|_{\omega_{d}}^{2} & =\sum_{k \in K_{0}} \frac{1}{\omega_{d}\left(1 / \lambda_{k}\right)}\left(\left|u_{1 k}\right|^{2}+\lambda_{k}^{2}\left|u_{0 k}\right|^{2}\right) \\
& \leq \sum_{k=0}^{\infty}\left[\varphi\left(\lambda_{k}\right)\right]^{1-4 \alpha} E_{k} \\
& =\sum_{h=0}^{\infty} \sum_{k \in B_{h}}\left[\varphi\left(\lambda_{k}\right)\right]^{1-4 \alpha} E_{k} .
\end{aligned}
$$

Now for every $k \in B_{h}$ it turns out that $n_{h} \leq \lambda_{k}<n_{h+1}$. From (5.17) and the fact that $4 \alpha<1$ it follows that

$$
\left[\varphi\left(\lambda_{k}\right)\right]^{1-4 \alpha} \leq 2^{h(1-4 \alpha)} \leq 2^{h}
$$

Keeping (5.14) into account, we deduce that

$$
\left\|\left(u_{0}, u_{1}\right)\right\|_{\omega_{d}}^{2} \leq \sum_{h=0}^{\infty} \sum_{k \in B_{h}}\left[\varphi\left(\lambda_{k}\right)\right]^{1-4 \alpha} E_{k} \leq \sum_{h=0}^{\infty} 2^{h} \sum_{k \in B_{h}} E_{k} \leq \sum_{h=0}^{\infty} 2^{h} \frac{E}{4^{h}}=2 E,
$$

which proves (5.12).

\section{A priori estimates for Kirchhoff equations}

In this section we derive a priori estimates for solutions to (1.1). Classical estimates are based on the usual Hamiltonian

$$
H(t):=\left|u^{\prime}(t)\right|^{2}+M\left(\left|A^{1 / 2} u(t)\right|^{2}\right),
$$

where

$$
M(x):=\int_{0}^{x} m(s) d s \quad \forall x \geq 0 .
$$

If $u(t)$ is a solution to (1.1)-(1.2) in some time-interval $[0, T]$, then the formal timederivative of $H(t)$ is given by

$$
H^{\prime}(t)=-4 \delta\left|A^{\sigma / 2} u^{\prime}(t)\right|^{2}
$$

and therefore

$$
\left|u^{\prime}(t)\right|^{2}+M\left(\left|A^{1 / 2} u(t)\right|^{2}\right)+4 \delta \int_{0}^{t}\left|A^{\sigma / 2} u^{\prime}(s)\right|^{2} d s=\left|u_{1}\right|^{2}+M\left(\left|A^{1 / 2} u_{0}\right|^{2}\right)
$$


for every $t \in[0, T]$. This is the classical way to obtain estimates both on $\left|u^{\prime}(t)\right|$ and on $\left|A^{1 / 2} u(t)\right|$. Unfortunately, things are not so simple under our assumptions.

A first problem comes from the lack of strict hyperbolicity when $\sigma>1 / 2$. In that case there is no guarantee that $M(x) \rightarrow+\infty$ as $x \rightarrow+\infty$, and hence (6.3) does not provide a bound on $\left|A^{1 / 2} u(t)\right|$, not even locally.

The second and even worse problem comes from regularity issues. Indeed, when computing the time-derivative of $H(t)$, we have to deal with terms such as $\left\langle u^{\prime}(t), A u(t)\right\rangle$, which are quite delicate in the case of weak solutions. In the literature this issue has been addressed in two different ways.

- If we write the term in the form $\left\langle A^{1 / 4} u^{\prime}(t), A^{3 / 4} u(t)\right\rangle$, then it makes sense for solutions living in the phase space $D\left(A^{3 / 4}\right) \times D\left(A^{1 / 4}\right)$, which is indeed the classical space both for Kirchhoff equations without dissipation, and for Kirchhoff equations with standard dissipation $(\sigma=0)$. This argument is probably hopeless in the case of less regular solutions. For example, when $\delta=0$ equation (6.2) seems to suggest that $H(t)$ is constant along trajectories, but as far as we know there is no rigorous proof of this fact for solutions with regularity less than $D\left(A^{3 / 4}\right) \times D\left(A^{1 / 4}\right)$.

- In the strongly dissipative case with $\sigma=1$, equality (6.3) seems to suggest that $A^{1 / 2} u^{\prime}(t)$ lies in $L^{2}((0, T), H)$. Therefore, if we write the term $\left\langle u^{\prime}(t), A u(t)\right\rangle$ in the form $\left\langle A^{1 / 2} u^{\prime}(t), A^{1 / 2} u(t)\right\rangle$, then it makes sense (at least almost everywhere) also for solutions in the energy space. This is the key point in the paper [28], in which Nishihara's theory [31] is extended to initial data in the energy space. Unfortunately, this heuristic argument strongly suggests that this approach is hopeless when $\sigma<1$.

Our assumptions are apparently too weak for both strategies. This leads us to follow a different path. As for the lack of coerciveness of $M(x)$, we introduce a modified Hamiltonian. As for the lack of regularity of solutions, when $\sigma \geq 1$ we exploit a strategy similar to the one described in the second point above. When $\sigma \in(0,1)$, we exploit the regularizing effect presented in section 3.2.

In the first a priori estimate we control $\left|A^{1 / 2} u(t)\right|$ in the supercritical case. We obtain an exponential bound, which is far from being optimal and could be improved to a polynomial bound with some additional effort. Nevertheless, any bound is enough in the sequel.

Proposition 6.1 (Supercritical dissipation - A priori bound). Let us consider problem (1.1)-(1.2) under the same assumptions of Theorem 2.1.

Let us assume that there exists a local solution $u(t)$, defined in the time-interval $[0, T]$ for some $T>0$, with the regularity prescribed by (3.2).

Then there exists two constants $K_{3}(\delta)$ and $K_{4}(\delta)$, depending only on $\delta$ (an in particular independent of $T$ and $u)$, such that

$$
\left|A^{1 / 2} u(t)\right|^{2} \leq K_{3}(\delta)\left(\left|u_{1}\right|^{2}+\left|u_{0}\right|^{2}+\left|A^{1 / 2} u_{0}\right|^{2}+M\left(\left|A^{1 / 2} u_{0}\right|^{2}\right)\right) \exp \left(K_{4}(\delta) t\right)
$$

for every $t \in[0, T]$. 
Proof Let us distinguish the cases $\sigma \geq 1$ and $1 / 2<\sigma<1$.

Case $\sigma \geq 1$ Let us set

$$
c(t):=m\left(\left|A^{1 / 2} u(t)\right|^{2}\right) .
$$

Let $\left\{u_{k}(t)\right\}$ denote the components of $u(t)$ with respect to the usual orthonormal system $\left\{e_{k}\right\}$, and let

$$
v_{n}(t):=\sum_{k=0}^{n} u_{k}(t) e_{k}, \quad c_{n}(t):=m\left(\left|A^{1 / 2} v_{n}(t)\right|^{2}\right) .
$$

It is easy to see that $v_{n}(t)$ is a solution to the linear problem

$$
v_{n}^{\prime \prime}(t)+2 \delta A^{\sigma} v_{n}^{\prime}(t)+c(t) A v_{n}(t)=0 .
$$

From the regularity assumption (3.2) it turns out that

$$
v_{n}^{\prime}(t) \rightarrow u^{\prime}(t), \quad A^{1 / 2} v_{n}(t) \rightarrow A^{1 / 2} u(t), \quad c_{n}(t) \rightarrow c(t),
$$

and the convergence is uniform in $[0, T]$ in all three cases (but in the sequel we need just pointwise convergence and uniform boundedness). Let us consider the modified approximated Hamiltonian

$$
H_{n}(t):=\left|v_{n}^{\prime}(t)\right|^{2}+\left|A^{1 / 2} v_{n}(t)\right|^{2}+M\left(\left|A^{1 / 2} v_{n}(t)\right|^{2}\right) .
$$

Its time-derivative is

$$
H_{n}^{\prime}(t)=-4 \delta\left|A^{\sigma / 2} v_{n}^{\prime}(t)\right|^{2}+2\left(c_{n}(t)-c(t)+1\right)\left\langle A^{1 / 2} v_{n}(t), A^{1 / 2} v_{n}^{\prime}(t)\right\rangle .
$$

Since $\sigma \geq 1$, it turns out that

$$
\left|A^{1 / 2} v_{n}^{\prime}(t)\right|^{2} \leq\left|A^{\sigma / 2} v_{n}^{\prime}(t)\right|^{2}+\left|v_{n}^{\prime}(t)\right|^{2},
$$

and hence

$$
\begin{aligned}
H_{n}^{\prime}(t) & \leq-4 \delta\left|A^{\sigma / 2} v_{n}^{\prime}(t)\right|^{2}+\frac{1}{4 \delta}\left(\left|c_{n}(t)-c(t)\right|+1\right)^{2}\left|A^{1 / 2} v_{n}(t)\right|^{2}+4 \delta\left|A^{1 / 2} v_{n}^{\prime}(t)\right|^{2} \\
& \leq \frac{1}{4 \delta}\left(\left|c_{n}(t)-c(t)\right|+1\right)^{2}\left|A^{1 / 2} v_{n}(t)\right|^{2}+4 \delta\left|v_{n}^{\prime}(t)\right|^{2} \\
& \leq\left(\frac{1}{4 \delta}\left(\left|c_{n}(t)-c(t)\right|+1\right)^{2}+4 \delta\right) H_{n}(t) .
\end{aligned}
$$

Integrating this differential inequality we obtain that

$$
\left|A^{1 / 2} v_{n}(t)\right|^{2} \leq H_{n}(t) \leq H_{n}(0) \exp \left(\frac{1}{4 \delta} \int_{0}^{t}\left(\left|c_{n}(s)-c(s)\right|+1\right)^{2} d s+4 \delta t\right) .
$$

Passing to the limit as $n \rightarrow+\infty$ we conclude that

$$
\left|A^{1 / 2} u(t)\right|^{2} \leq\left(\left|u_{1}\right|^{2}+\left|A^{1 / 2} u_{0}\right|^{2}+M\left(\left|A^{1 / 2} u_{0}\right|^{2}\right)\right) \exp \left(\frac{t}{4 \delta}+4 \delta t\right),
$$

which proves $(6.4)$ in the case $\sigma \geq 1$ with $K_{3}(\delta):=1$ and $K_{4}(\delta):=4 \delta+(4 \delta)^{-1}$. 
Case $1 / 2<\sigma<1 \quad$ Let us consider $u(t)$ as a solution to the linear equation (1.10) with the coefficient $c(t)$ defined as in (6.5). Let us consider the Hamiltonian $H(t)$ defined in (6.1). Since $\sigma \in(1 / 2,1)$, from Theorem $\mathrm{C}$ we know that $u^{\prime}(t)$ is continuous with values in $D\left(A^{1 / 2}\right)$ for every $t \in(0, T]$, or at least for every $t \in\left(0, S_{0}\right) \cup\left(S_{0}, T\right]$ for some $S_{0} \in(0, T)$.

This is enough to justify rigorously the calculation leading to (6.2). Indeed, the terms such as $\left\langle u^{\prime}(t), A u(t)\right\rangle$ which appear during the computation of $H^{\prime}(t)$ can be interpreted in the form $\left\langle A^{1 / 2} u^{\prime}(t), A^{1 / 2} u(t)\right\rangle$, and hence they are well-defined when both $u(t)$ and $u^{\prime}(t)$ are continuous with values in $D\left(A^{1 / 2}\right)$. This proves that (6.2) holds true for every $t \in(0, T]$, or at least for every $t \in\left(0, S_{0}\right) \cup\left(S_{0}, T\right]$. Since $H(t)$ is continuous because of the regularity assumption (3.2), this is enough to conclude that (6.3) holds true for every $t \in[0, T]$.

Let us consider now the modified Hamiltonian

$$
\widehat{H}(t)=H(t)+\delta^{2}|u(t)|^{2}+\delta^{2}\left|A^{1 / 2} u(t)\right|^{2}+\delta\left\langle A^{1-\sigma} u(t), u^{\prime}(t)\right\rangle,
$$

which is well-defined in the range $1 / 2 \leq \sigma \leq 1$. In this range it turns out that

$$
\begin{aligned}
\left|\delta\left\langle A^{1-\sigma} u(t), u^{\prime}(t)\right\rangle\right| & \leq \delta\left|A^{1-\sigma} u(t)\right| \cdot\left|u^{\prime}(t)\right| \\
& \leq \frac{1}{2}\left|u^{\prime}(t)\right|^{2}+\frac{\delta^{2}}{2}\left|A^{1-\sigma} u(t)\right|^{2} \\
& \leq \frac{1}{2}\left|u^{\prime}(t)\right|^{2}+\frac{\delta^{2}}{2}\left|A^{1 / 2} u(t)\right|^{2}+\frac{\delta^{2}}{2}|u(t)|^{2},
\end{aligned}
$$

and therefore

$$
\widehat{H}(0) \leq 2\left|u_{1}\right|^{2}+2 \delta^{2}\left|u_{0}\right|^{2}+2 \delta^{2}\left|A^{1 / 2} u_{0}\right|^{2}+M\left(\left|A^{1 / 2} u_{0}\right|^{2}\right)
$$

and

$$
\frac{\delta^{2}}{2}\left|A^{1 / 2} u(t)\right|^{2} \leq \widehat{H}(t) \quad \forall t \in[0, T] .
$$

Now we claim that $\widehat{H}(t)$ is time-differentiable where $H(t)$ is time-differentiable, namely in $(0, T]$ or in $\left(0, S_{0}\right) \cup\left(S_{0}, T\right]$, and its time-derivative is

$$
\widehat{H}^{\prime}(t)=-4 \delta\left|A^{\sigma / 2} u^{\prime}(t)\right|^{2}+2 \delta^{2}\left\langle u(t), u^{\prime}(t)\right\rangle+\delta\left|A^{(1-\sigma) / 2} u^{\prime}(t)\right|^{2}-\delta c(t)\left|A^{1-\sigma / 2} u(t)\right|^{2} .
$$

Indeed, in addition to the terms due to $H^{\prime}(t)$, now the most dangerous term is the last one. In order to show that this term is well-defined, we consider the same three cases as in the statement of Theorem C. In the first case, the term is well-defined because of the regularity of $u(t)$. In the second case, it is well-defined because $c(t) \equiv 0$ in $[0, T]$. In the third case, it is well-defined in $\left[0, S_{0}\right]$ because $c(t) \equiv 0$ in that interval, and it is well-defined in $\left(S_{0}, T\right]$ because of the regularity of $u(t)$ in that interval.

In order to estimate $\widehat{H}^{\prime}(t)$, we observe that

$$
2 \delta^{2}\left\langle u(t), u^{\prime}(t)\right\rangle \leq \delta^{2}|u(t)|^{2}+\delta^{2}\left|u^{\prime}(t)\right|^{2}
$$


and, since $\sigma \geq 1 / 2$,

$$
\delta\left|A^{(1-\sigma) / 2} u^{\prime}(t)\right|^{2} \leq \delta\left|A^{\sigma / 2} u^{\prime}(t)\right|^{2}+\delta\left|u^{\prime}(t)\right|^{2}
$$

It follows that

$$
\widehat{H}^{\prime}(t) \leq \delta^{2}|u(t)|^{2}+\left(\delta^{2}+\delta\right)\left|u^{\prime}(t)\right|^{2}
$$

Now we estimate $\left|u^{\prime}(t)\right|$ by means of (6.3), and we estimate $|u(t)|$ by observing that

$$
|u(t)| \leq\left|u_{0}\right|+\int_{0}^{t}\left|u^{\prime}(s)\right| d s \leq\left|u_{0}\right|+t H(0)^{1 / 2}
$$

and hence

$$
|u(t)|^{2} \leq 2\left|u_{0}\right|^{2}+2 H(0) t^{2}
$$

It follows that

$$
\widehat{H}^{\prime}(t) \leq 2 \delta^{2}\left|u_{0}\right|^{2}+2 \delta^{2} H(0) t^{2}+\left(\delta^{2}+\delta\right) H(0)
$$

for every $t \in(0, T]$, or at least for every $t \in\left(0, S_{0}\right) \cup\left(S_{0}, T\right]$. Since $\widehat{H}(t)$ is continuous in $[0, T]$, a simple integration gives that

$$
\widehat{H}(t) \leq \widehat{H}(0)+2 \delta^{2}\left|u_{0}\right|^{2} t+\left(\delta^{2}+\delta\right) H(0) t+2 \delta^{2} H(0) \frac{t^{3}}{3} \quad \forall t \in[0, T] .
$$

Now we estimate $\widehat{H}(0)$ as in (6.6) and powers of $t$ with $e^{t}$. Keeping (6.7) into account, we obtain an estimate of the form $(6.4)$, with $K_{4}(\delta):=1$ and a suitable $K_{3}(\delta)$.

Finally, if we want $K_{3}(\delta)$ and $K_{4}(\delta)$ to be the same in the case $\sigma \geq 1$ and in the case $1 / 2 \leq \sigma \leq 1$, it is enough to take for each of them the maximum of the values obtained in the two ranges.

Our second a priori estimate concerns the subcritical case $\sigma \in(0,1 / 2]$. We state the result under the assumptions we need in the sequel, even if slightly different from those of Theorem 2.2 .

Proposition 6.2 (Subcritical dissipation - A priori bound). Let us consider equation (1.1) under the following assumptions:

- A is a self-adjoint nonnegative operator on a separable Hilbert space $H$,

- $m:[0,+\infty) \rightarrow[0,+\infty)$ satisfies the strict hyperbolicity assumption (1.4),

- $\sigma \in(0,1 / 2]$ and $\delta>0$ are two real numbers.

Let us assume that there exists a local solution $u(t)$, defined in the time-interval $[0, T]$ for some $T>0$, with the regularity prescribed by (3.2). Let us assume in addition that the continuity modulus of the function $t \rightarrow m\left(\left|A^{1 / 2} u(t)\right|^{2}\right)$ satisfies the assumptions of Theorem B, and in particular inequality (3.7) with $\Lambda_{\infty}$ defined by (3.8).

Then it turns out that

$$
\left|u^{\prime}(t)\right|^{2}+\left|A^{1 / 2} u(t)\right|^{2} \leq \max \left\{1, \frac{1}{\mu_{1}}\right\}\left(\left|u_{1}\right|^{2}+M\left(\left|A^{1 / 2} u_{0}\right|^{2}\right)\right) \quad \forall t \in[0, T] .
$$


Proof Let us consider $u(t)$ as a solution to the linear equation (1.10) with the coefficient $c(t)$ defined as in (6.5). This coefficient is bounded from below by $\mu_{1}$ because of the strict hyperbolicity assumption, and bounded from above by some $\mu_{2}$ because of its continuity. Moreover, we assumed that the continuity modulus of $c(t)$ satisfies (3.7). As a consequence, we are in the assumptions of Theorem B and Theorem D, from which we deduce further regularity of $u^{\prime}(t)$ in $(0, T]$.

This is enough to justify rigorously the computation of the time-derivative of $H(t)$ leading to (6.2), which therefore holds true for every $t \in(0, T]$. Since $H(t)$ is continuous, equality (6.3) holds true for every $t \in[0, T]$.

Finally, the strict hyperbolicity assumption (1.4) implies that $M(x) \geq \mu_{1} x$ for every $x \geq 0$. At this point, (6.8) follows from (6.3).

\section{$7 \quad$ Proof of main results}

We are now ready to prove Theorem 2.1 and Theorem 2.2. Actually, we prove two stronger results, which we state below.

The first one is a stronger version of Theorem 2.1 in interpolation spaces.

Theorem 7.1 (Supercritical dissipation). Let us consider problem (1.1)-(1.2) under the same assumptions of Theorem 2.1.

Let us assume in addition that $\left(u_{0}, u_{1}\right) \in V_{\omega_{d}}$ for some continuity modulus $\omega_{d}(x)$.

Then the problem admits at least one global solution

$$
u \in C^{0}\left([0,+\infty), D\left(A^{1 / 2}\right)\right) \cap C^{1}([0,+\infty), H)
$$

such that

$$
\left(u, u^{\prime}\right) \in C^{0}\left([0,+\infty), V_{\omega_{d}}\right) .
$$

From Proposition 5.6, applied with $\alpha=0$, we know that every pair of initial conditions $\left(u_{0}, u_{1}\right) \in D\left(A^{1 / 2}\right) \times H$ lies in $V_{\omega_{d}}$ for a suitable continuity modulus $\omega_{d}(x)$ depending on $\left(u_{0}, u_{1}\right)$. As a consequence, Theorem 7.1 above implies Theorem 2.1.

The second result is a stronger version of Theorem 2.2.

Theorem 7.2 (Subcritical dissipation). Let us consider problem (1.1)-(1.2) under the same assumptions of Theorem 2.2.

Let us assume in addition that $\left(u_{0}, u_{1}\right) \in V_{\omega_{d}}$ for some continuity modulus $\omega_{d}(x)$ satisfying (5.9) through (5.11) with the same $\alpha \in[0,1 / 4)$ which appears in (2.5).

Then the following conclusions hold true.

(1) (Local existence) There exists $T>0$ such that problem (1.1)-(1.2) admits at least one local solution

$$
u \in C^{0}\left([0, T], D\left(A^{1 / 2}\right)\right) \cap C^{1}([0, T], H)
$$

such that

$$
\left(u, u^{\prime}\right) \in C^{0}\left([0, T], V_{\omega_{d}}\right)
$$


(2) (Alternative) Every local solution satisfying (7.3) and (7.4) can be continued to a solution with the same regularity defined on a maximal time-interval $\left[0, T_{*}\right)$, and either $T_{*}=+\infty$ or

$$
\limsup _{t \rightarrow T_{*}^{-}}\left\|\left(u(t), u^{\prime}(t)\right)\right\|_{\omega_{d}}=+\infty
$$

(3) (Global existence) There exists $\varepsilon_{1}>0$, independent of $\omega_{d}(x)$ (provided of course that (5.9) through (5.11) are satisfied), with the following property. If the initial conditions $\left(u_{0}, u_{1}\right)$ satisfy

$$
\left|u_{1}\right|^{2}+\left|A^{1 / 2} u_{0}\right|^{2}+\left\|\left(u_{0}, u_{1}\right)\right\|_{\omega_{d}}^{2} \leq \varepsilon_{1},
$$

then every local solution satisfying (7.3) and (7.4) can be continued to a global solution with the regularity (7.1) and (7.2).

From Proposition 5.6, applied with the same value of $\alpha$ for which (2.5) holds true, we know that every pair of initial conditions $\left(u_{0}, u_{1}\right) \in D\left(A^{\alpha+1 / 2}\right) \times D\left(A^{\alpha}\right)$ lies in $V_{\omega_{d}}$ for a suitable continuity modulus $\omega_{d}(x)$ satisfying (5.9) through (5.12). As a consequence, the local existence statement of Theorem 7.2 above implies the local existence statement of Theorem 2.2. Up to now, we did not exploit (5.12), which only comes into play in the global existence statement.

If in addition the pair $\left(u_{0}, u_{1}\right)$ is small in the sense of (2.6), then from (5.12) it follows that $\left(u_{0}, u_{1}\right)$ is small also in the sense of (7.6) with $\varepsilon_{1}=2 \varepsilon_{0}$. Since $\varepsilon_{1}$ does not depend on $\omega_{d}(x)$, the global existence statement of Theorem 7.2 above implies the global existence statement of Theorem 2.2.

In conclusion, our main results are true if we prove Theorem 7.1 and Theorem 7.2.

\section{Proof of Theorem 7.1}

Local existence Let us set

$$
M_{0}:=\left|A^{1 / 2} u_{0}\right|^{2}+1, \quad \mu_{2}:=\max _{x \in\left[0, M_{0}\right]} m(x), \quad m_{*}(x):=m\left(\min \left\{x, M_{0}\right\}\right) .
$$

In such a way, $m_{*}(x)$ is a nonnegative continuous function, which is bounded from above by $\mu_{2}$, and coincides with $m(x)$ for every $x \in\left[0, M_{0}\right]$. Moreover, the continuity modulus of $m_{*}(x)$ in $[0,+\infty)$ coincides with the continuity modulus of $m(x)$ in $\left[0, M_{0}\right]$, which we denote by $\omega_{m}(x)$.

We claim that problem (1.1)-(1.2), with $m_{*}(x)$ instead of $m(x)$, admits at least one local solution $u_{*}(t)$, defined in some time-interval $[0, T]$, with regularity

$$
u_{*} \in C^{0}\left([0, T], D\left(A^{1 / 2}\right)\right) \cap C^{1}([0, T], H),
$$

and

$$
\left(u_{*}, u_{*}^{\prime}\right) \in C^{0}\left([0, T], V_{\omega_{d}}\right) .
$$


If we prove this claim, then $u_{*}(t)$ turns out to be also a solution to the original problem in a possibly smaller time-interval. Indeed, since $\left|A^{1 / 2} u_{*}(t)\right|^{2}$ is a continuous function and $\left|A^{1 / 2} u_{*}(0)\right|^{2}=\left|A^{1 / 2} u_{0}\right|^{2}<M_{0}$, there exists $T_{1} \in(0, T]$ such that $\left|A^{1 / 2} u_{*}(t)\right|^{2} \leq M_{0}$ for every $t \in\left[0, T_{1}\right]$. As a consequence

$$
m_{*}\left(\left|A^{1 / 2} u_{*}(t)\right|^{2}\right)=m\left(\left|A^{1 / 2} u_{*}(t)\right|^{2}\right) \quad \forall t \in\left[0, T_{1}\right],
$$

and hence $u_{*}(t)$ is also a solution to the original problem in $\left[0, T_{1}\right]$.

In order to prove the existence of $u_{*}(t)$, we exploit a fixed point argument. Let us choose a positive number $T$, and a real number $\nu \geq 1$ satisfying (3.3). Let us set

$$
\omega_{c}(x):=\omega_{m}\left(3 K_{1}\left(\delta, \mu_{2}\right) e^{\left(1+\mu_{2}\right) \nu T} \cdot\left\|\left(u_{0}, u_{1}\right)\right\|_{\omega_{d}}^{2} \cdot \omega_{d}(x)\right),
$$

where $K_{1}\left(\delta, \mu_{2}\right)$ is the constant of Theorem $\mathrm{A}$. Let $\mathbb{X}$ be the space of all continuous functions $c:[0, T] \rightarrow\left[0, \mu_{2}\right]$ with continuity modulus equal to $\omega_{c}(x)$. Due to Ascoli's Theorem, the space $\mathbb{X}$ turns out to be a compact and convex subset of the Banach space of all continuous functions $c:[0, T] \rightarrow \mathbb{R}$, endowed with the sup norm.

For every $c \in \mathbb{X}$, we consider the solution $u(t)$ to the linear problem (1.10)-(1.2), and then we define a function $\Phi(c):[0, T] \rightarrow \mathbb{R}$ as

$$
[\Phi(c)](t):=m_{*}\left(\left|A^{1 / 2} u(t)\right|^{2}\right) \quad \forall t \in[0, T] .
$$

We claim that $\Phi$ is a continuous map from $\mathbb{X}$ to $\mathbb{X}$. If we prove this claim, then Schauder's fixed point theorem implies that $\Phi$ admits at least one fixed point $c(t)$. The corresponding solution to the linear problem is the solution $u_{*}(t)$ we were looking for, and its regularity (7.1) follows from Theorem A, while its regularity (7.2) follows from Proposition 5.2.

So let us verify the properties of $\Phi$. First of all, we need to show that $\Phi(c) \in \mathbb{X}$ for every $c \in \mathbb{X}$. The fact that $[\Phi(c)](t) \in\left[0, \mu_{2}\right]$ for every $t \in[0, T]$ follows from the bounds on $m_{*}(x)$. The main point is proving that the map $t \rightarrow[\Phi(c)](t)$ has continuity modulus equal to $\omega_{c}(x)$. To this end, we begin by applying Proposition 5.2, from which we deduce that $\left(u(t), u^{\prime}(t)\right) \in V_{\omega_{d}}$ for every $t \in[0, T]$ and (5.1) holds true. This allows us to apply Proposition 5.5, from which we deduce that

$$
\left.|| A^{1 / 2} u(a)\right|^{2}-\left|A^{1 / 2} u(b)\right|^{2} \mid \leq 3 K_{1}\left(\delta, \mu_{2}\right) e^{\left(1+\mu_{2}\right) \nu T} \cdot\left\|\left(u_{0}, u_{1}\right)\right\|_{\omega_{d}}^{2} \cdot \omega_{d}(|a-b|)
$$

for every $a$ and $b$ in $[0, T]$. Since $m_{*}(x)$ has continuity modulus $\omega_{m}(x)$, we conclude that

$$
\begin{aligned}
|[\Phi(c)](a)-[\Phi(c)](b)| & =\left|m_{*}\left(\left|A^{1 / 2} u(a)\right|^{2}\right)-m_{*}\left(\left|A^{1 / 2} u(b)\right|^{2}\right)\right| \\
& \leq \omega_{m}\left(\left.|| A^{1 / 2} u(a)\right|^{2}-\left|A^{1 / 2} u(b)\right|^{2} \mid\right) \\
& \leq \omega_{m}\left(3 K_{1}\left(\delta, \mu_{2}\right) e^{\left(1+\mu_{2}\right) \nu T} \cdot\left\|\left(u_{0}, u_{1}\right)\right\|_{\omega_{d}}^{2} \cdot \omega_{d}(|a-b|)\right) \\
& =\omega_{c}(|a-b|)
\end{aligned}
$$

for every $a$ and $b$ in $[0, T]$, which completes the proof that $\Phi(c) \in \mathbb{X}$.

Now that we know that $\Phi$ maps $\mathbb{X}$ to $\mathbb{X}$, its continuity with respect to the sup norm is a simple application of Theorem 4.1. This completes the proof of local existence. 
Alternative Every local solution to (1.1)-(1.2) satisfying (7.8) and (7.9) can be continued to a maximal solution $u(t)$ defined in some time-interval $\left[0, T_{*}\right)$, and such that

$$
\begin{gathered}
u \in C^{0}\left(\left[0, T_{*}\right), D\left(A^{1 / 2}\right)\right) \cap C^{1}\left(\left[0, T_{*}\right), H\right), \\
\left(u, u^{\prime}\right) \in C^{0}\left(\left[0, T_{*}\right), V_{\omega_{d}}\right) .
\end{gathered}
$$

We prove that either $T_{*}=+\infty$ or

$$
\limsup _{t \rightarrow T_{*}^{-}}\left\|\left(u(t), u^{\prime}(t)\right)\right\|_{\omega_{d}}^{2}=+\infty
$$

To this end, let us assume by contradiction that $T_{*}<+\infty$ and (7.13) is false, so that

$$
L_{0}:=\sup _{t \in\left[0, T_{*}\right)}\left\|\left(u(t), u^{\prime}(t)\right)\right\|_{\omega_{d}}^{2}<+\infty .
$$

We claim that $u(t)$ can be extended to the closed interval $\left[0, T_{*}\right]$ in such a way that

$$
u \in C^{0}\left(\left[0, T_{*}\right], D\left(A^{1 / 2}\right)\right) \cap C^{1}\left(\left[0, T_{*}\right], H\right),
$$

with in addition

$$
\left(u, u^{\prime}\right) \in C^{0}\left(\left[0, T_{*}\right], V_{\omega_{d}}\right) .
$$

If we prove this claim, then we can use $\left(u\left(T_{*}\right), u^{\prime}\left(T_{*}\right)\right)$ as a new initial condition and extend the solution beyond $T_{*}$, thus contradicting its maximality.

In order to prove the claim, we apply Proposition 5.5 in every closed interval $[0, T] \subseteq$ $\left[0, T_{*}\right)$. We deduce that the function $t \rightarrow\left|A^{1 / 2} u(t)\right|^{2}$ is continuous in $[0, T]$ with continuity modulus equal to $3 L_{0} \omega_{d}(x)$. Since the continuity modulus does not depend on $T$, the function is continuous with the same continuity modulus in $\left[0, T_{*}\right)$, and in particular it is bounded in the same interval. As a consequence, the function $t \rightarrow c(t):=m\left(\left|A^{1 / 2} u(t)\right|^{2}\right)$ is uniformly continuous in $\left[0, T_{*}\right)$, and hence it can be extended to a continuous function defined in the closed interval $\left[0, T_{*}\right]$.

The function $c(t)$, in the closed interval $\left[0, T_{*}\right]$, is nonnegative and bounded from above. Therefore, from Theorem A and Proposition 5.2 it follows that the linear problem (1.10)-(1.2) has a unique solution in the closed interval $\left[0, T_{*}\right]$ with the regularity $(7.15)$ and (7.16), which is the required extension of $u$.

Global existence Let $u(t)$ be a local solution defined in a maximal interval $\left[0, T_{*}\right)$. Let us assume by contradiction that $T_{*}<+\infty$. Due to the a priori estimate of Proposition 6.1, we know that $\left|A^{1 / 2} u(t)\right|$ is uniformly bounded in $\left[0, T_{*}\right)$, hence also $c(t)$ is uniformly bounded from above in $\left[0, T_{*}\right)$ by some constant $\widehat{\mu}_{2}$. Therefore, from Proposition 5.2 we deduce that

$$
\left\|\left(u(t), u^{\prime}(t)\right)\right\|_{\omega_{d}}^{2} \leq K_{1}\left(\delta, \widehat{\mu}_{2}\right) e^{\left(1+\widehat{\mu}_{2}\right) \nu t} \cdot\left\|\left(u_{0}, u_{1}\right)\right\|_{\omega_{d}}^{2} \quad \forall t \in\left[0, T_{*}\right) .
$$

Passing to the limit as $t \rightarrow T_{*}^{-}$we contradict (7.13). 


\section{Proof of Theorem 7.2}

Local existence The argument is similar to the proof of Theorem 7.1. Once again, we define $M_{0}, \mu_{2}, m_{*}(x)$ as in (7.7), and we look for a solution $u_{*}(t)$ to the modified problem, which once again turns out to be a solution to the original problem in a possibly smaller time-interval. We remark that in this case $m_{*}(x)$ is bounded from below by $\mu_{1}$ due to the strict hyperbolicity assumption (1.4).

The existence of $u_{*}(t)$ follows again from Schauder's fixed point theorem. This time we set

$$
\omega_{c}(x):=\omega_{m}\left(6 K_{2}\left(\delta, \mu_{1}, \mu_{2}\right) \cdot\left\|\left(u_{0}, u_{1}\right)\right\|_{\omega_{d}}^{2} \cdot \omega_{d}(x)\right),
$$

where $K_{2}\left(\delta, \mu_{1}, \mu_{2}\right)$ is the constant of Theorem B. First of all, we prove that

$$
\lim _{x \rightarrow 0^{+}} \frac{\omega_{m}\left(\omega_{d}(x)\right)}{x^{1-2 \sigma}}=0
$$

This is trivial if $\sigma=1 / 2$. If $\sigma>1 / 2$, the value $\alpha$ for which (2.5) holds true is positive. Therefore, we can write

$$
\frac{\omega_{m}\left(\omega_{d}(x)\right)}{x^{1-2 \sigma}}=\left(\frac{\left[\omega_{m}\left(\omega_{d}(x)\right)\right]^{4 \alpha}}{\left[\omega_{d}(x)\right]^{1-2 \sigma}}\right)^{1 /(4 \alpha)}\left(\frac{\omega_{d}(x)}{x^{4 \alpha}}\right)^{(1-2 \sigma) /(4 \alpha)},
$$

and observe that the first term is bounded because of (2.5), while the second term tends to 0 as $x \rightarrow 0^{+}$because of (5.11).

From (7.17) and (2.3) it follows that

$$
\lim _{x \rightarrow 0^{+}} \frac{\omega_{c}(x)}{x^{1-2 \sigma}}=0
$$

and hence there exists $\nu>0$ such that

$$
4 \delta^{2} \mu_{1} \geq\left[\lambda^{1-2 \sigma} \omega_{c}\left(\frac{1}{\lambda}\right)\right]^{2}+2 \delta\left[\lambda^{1-2 \sigma} \omega_{c}\left(\frac{1}{\lambda}\right)\right] \quad \forall \lambda \geq \nu .
$$

Given this value of $\nu$, we finally set

$$
T:=\frac{\log 2}{\left(1+\mu_{2}\right) \nu} .
$$

Now we proceed again in analogy with the proof of Theorem 7.1. We consider the space $\mathbb{X}$ of all functions $c:[0, T] \rightarrow\left[\mu_{1}, \mu_{2}\right]$ with continuity modulus $\omega_{c}(x)$, and for every $c \in \mathbb{X}$ we define $\Phi(c):[0, T] \rightarrow \mathbb{R}$ as in (7.10). The solution $u(t)$ to the linear problem (1.10)-(1.2) needed in the definition is now provided by Theorem B, whose assumptions are satisfied because $\omega_{c}(x)$ satisfies (7.18).

Once again, the main point is proving that the continuity modulus of the map $t \rightarrow[\Phi(c)](t)$ is $\omega_{c}(x)$. To this end, we begin by applying Proposition 5.3, from which 
we deduce that $\left(u, u^{\prime}\right) \in C^{0}\left([0, T], V_{\omega_{d}}\right)$ and (5.2) holds true. Therefore, from (7.20) it follows that

$$
\left\|\left(u(t), u^{\prime}(t)\right)\right\|_{\omega_{d}}^{2} \leq 2 K_{2}\left(\delta, \mu_{1}, \mu_{2}\right)\left\|\left(u_{0}, u_{1}\right)\right\|_{\omega_{d}}^{2} \quad \forall t \in[0, T]
$$

This allows us to apply Proposition 5.5, from which we deduce that

$$
\left.|| A^{1 / 2} u(a)\right|^{2}-\left|A^{1 / 2} u(b)\right|^{2} \mid \leq 6 K_{2}\left(\delta, \mu_{1}, \mu_{2}\right)\left\|\left(u_{0}, u_{1}\right)\right\|_{\omega_{d}}^{2} \cdot \omega_{d}(|a-b|)
$$

for every $a$ and $b$ in $[0, T]$. At this point we conclude as in the proof of Theorem 7.1.

Alternative We argue as in the corresponding paragraph of the proof of Theorem 7.1. Assuming by contradiction that $T_{*}<+\infty$ and (7.14) holds true, the key point is extending $c(t)$ to the closed interval $\left[0, T_{*}\right]$ in such a way that the assumptions of Theorem $\mathrm{B}$ and Proposition 5.3 are satisfied, so that also the solution $u(t)$ can be continued to $\left[0, T_{*}\right]$ with the required regularity.

In analogy with the proof of Theorem 7.1, from Proposition 5.5 we deduce that the function $t \rightarrow\left|A^{1 / 2} u(t)\right|^{2}$ is uniformly continuous in $\left[0, T_{*}\right)$, with continuity modulus equal to $3 L_{0} \omega_{d}(x)$. As a consequence, the function $t \rightarrow c(t)$ is uniformly continuous in $\left[0, T_{*}\right)$, with continuity modulus $\omega_{m}\left(3 L_{0} \omega_{d}(x)\right)$, and hence it can be extended to the closed interval $\left[0, T_{*}\right]$ with the same continuity modulus. On the other hand, this continuity modulus satisfies (3.7) with $\Lambda_{\infty}=0$ (same proof as in the case of $\omega_{c}(x)$, just with a different constant). Therefore, the assumptions of Theorem B are satisfied.

Global existence Let us consider a new continuity modulus defined by

$$
\widehat{\omega}_{c}(x):=\omega_{m}\left(6 L_{1} x^{4 \alpha}\right),
$$

where $L_{1}>0$ is a parameter to be chosen. We claim that $\widehat{\omega}_{c}(x)$ satisfies (7.19) with $\nu=1$, namely

$$
4 \delta^{2} \mu_{1} \geq\left[\lambda^{1-2 \sigma} \widehat{\omega}_{c}\left(\frac{1}{\lambda}\right)\right]^{2}+2 \delta\left[\lambda^{1-2 \sigma} \widehat{\omega}_{c}\left(\frac{1}{\lambda}\right)\right] \quad \forall \lambda \geq 1
$$

provided that $L_{1}$ is small enough. If $\sigma=1 / 2$, this is almost obvious. If $\sigma<1 / 2$, the value $\alpha$ for which (2.5) holds true is positive, and hence

$$
R:=\sup _{y \in(0,1]} \frac{\omega_{m}\left(y^{4 \alpha}\right)}{y^{1-2 \sigma}}=\sup _{z \in(0,1]}\left(\frac{\left[\omega_{m}(z)\right]^{4 \alpha}}{z^{1-2 \sigma}}\right)^{1 / 4 \alpha}<+\infty .
$$

Therefore, when $6 L_{1} \leq 1$ it follows that

$$
\sup _{x \in(0,1]} \frac{\widehat{\omega}_{c}(x)}{x^{1-2 \sigma}}=\sup _{x \in(0,1]} \frac{\omega_{m}\left(\left[\left(6 L_{1}\right)^{1 /(4 \alpha)} x\right]^{4 \alpha}\right)}{\left[\left(6 L_{1}\right)^{1 /(4 \alpha)} x\right]^{1-2 \sigma}} \cdot\left(6 L_{1}\right)^{(1-2 \sigma) /(4 \alpha)} \leq R \cdot\left(6 L_{1}\right)^{(1-2 \sigma) /(4 \alpha)}
$$


which implies (7.21) when $L_{1}$ is small enough.

Now let us choose $L_{1}>0$ so that (7.21) holds true, let us set

$$
\widehat{\mu}_{2}:=\max \left\{m(x): x \in\left[0, L_{1}\right]\right\}
$$

and let us assume that $\varepsilon_{1}$ in (7.6) is small enough so that

$$
K_{2}\left(\delta, \mu_{1}, \widehat{\mu}_{2}\right)\left\|\left(u_{0}, u_{1}\right)\right\|_{\omega_{d}}^{2}+\max \left\{1, \frac{1}{\mu_{1}}\right\}\left(\left|u_{1}\right|^{2}+M\left(\left|A^{1 / 2} u_{0}\right|^{2}\right)\right) \leq L_{1}
$$

where $K_{2}\left(\delta, \mu_{1}, \widehat{\mu}_{2}\right)$ is once again the constant of Theorem B. We point out that this smallness condition on $\varepsilon_{1}$ is independent of $\omega_{d}(x)$.

For any such initial condition, let us consider a local solution $u(t)$ to $(1.1)-(1.2)$, defined on a maximal interval $\left[0, T_{*}\right)$, with the regularity prescribed by $(7.11)$ and $(7.12)$.

We claim that

$$
\left\|\left(u(t), u^{\prime}(t)\right)\right\|_{\omega_{d}}^{2} \leq 2 L_{1} \quad \forall t \in\left[0, T_{*}\right) .
$$

Since this contradicts (7.5), the only possibility left by the alternative is that $T_{*}=$ $+\infty$, which means that $u(t)$ is a global solution. In order to prove (7.23), we introduce

$$
S:=\sup \left\{t \in\left[0, T_{*}\right):\left\|\left(u(\tau), u^{\prime}(\tau)\right)\right\|_{\omega_{d}} \leq 2 L_{1} \quad \forall \tau \in[0, t]\right\},
$$

so that (7.23) is now equivalent to proving that $S=T_{*}$.

From (7.22) it follows that $\left\|\left(u(0), u^{\prime}(0)\right)\right\|_{\omega_{d}} \leq L_{1}<2 L_{1}$ (because $K_{2}\left(\delta, \mu_{1}, \widehat{\mu}_{2}\right) \geq 1$, as already observed after Theorem B). Therefore, $S$ is the supremum of a nonempty set, and $S>0$ because the function $t \rightarrow\left\|\left(u(t), u^{\prime}(t)\right)\right\|_{\omega_{d}}$ is continuous. Moreover, the maximality of $S$ implies that either $S=T_{*}$, or

$$
\left\|\left(u(S), u^{\prime}(S)\right)\right\|_{\omega_{d}}^{2}=2 L_{1} .
$$

If we prove that (7.24) does not hold, then automatically $S=T_{*}$. So let us assume by contradiction that $S<T_{*}$. From our definition of $S$ it follows that

$$
\left\|\left(u(t), u^{\prime}(t)\right)\right\|_{\omega_{d}}^{2} \leq 2 L_{1} \quad \forall t \in[0, S] .
$$

Therefore, from Proposition 5.5 we obtain that the function $t \rightarrow\left|A^{1 / 2} u(t)\right|^{2}$ has continuity modulus $6 L_{1} \omega_{d}(x)$ in $[0, S]$. As a consequence, the function

$$
c(t):=m\left(\left|A^{1 / 2} u(t)\right|^{2}\right)
$$

has continuity modulus $\omega_{m}\left(6 L_{1} \omega_{d}(x)\right)$ in $[0, S]$, and this continuity modulus is less than or equal to $\widehat{\omega}_{c}(x)$ because of (5.10). Thus the continuity modulus of $c(t)$ satisfies (3.7), and hence we can apply Proposition 6.2 and deduce that (6.8) holds true for every $t \in[0, S]$. In particular, $\left|A^{1 / 2} u(t)\right|^{2} \leq L_{1}$ and hence $c(t) \leq \widehat{\mu}_{2}$ for every $t \in[0, S]$.

Now we estimate the (semi)norm of $\left(u(t), u^{\prime}(t)\right)$ in $V_{\omega_{d}}$. To this end, we interpret $u(t)$ as a solution to the linear equation (1.10) with coefficient $c(t)$ given by (7.25). Since $c(t)$ 
has continuity modulus (less than or equal to) $\widehat{\omega}_{c}(x)$, the assumptions of Proposition 5.3 are satisfied with $\nu:=1$. As for low-frequency components, from (5.4) with $\nu=1$ it follows that

$$
\begin{aligned}
\left\|\left(u_{\nu=1,-}(t), u_{\nu=1,-}^{\prime}(t)\right)\right\|_{\omega_{d}}^{2} & \leq \frac{1}{\omega_{d}(1)}\left(\left|u_{\nu=1,-}^{\prime}(t)\right|^{2}+\left|A^{1 / 2} u_{\nu=1,-}(t)\right|^{2}\right) \\
& \leq \frac{1}{\omega_{d}(1)}\left(\left|u^{\prime}(t)\right|^{2}+\left|A^{1 / 2} u(t)\right|^{2}\right) .
\end{aligned}
$$

Keeping into account that $\omega_{d}(1)=1$, from the a priori estimate of Proposition 6.2 we deduce that

$$
\left\|\left(u_{\nu=1,-}(t), u_{\nu=1,-}^{\prime}(t)\right)\right\|_{\omega_{d}}^{2} \leq \max \left\{1, \frac{1}{\mu_{1}}\right\}\left(\left|u_{1}\right|^{2}+M\left(\left|A^{1 / 2} u_{0}\right|^{2}\right)\right) .
$$

As for high-frequency components, from (5.3) it follows that

$$
\left\|\left(u_{\nu=1,+}(t), u_{\nu=1,+}^{\prime}(t)\right)\right\|_{\omega_{d}}^{2} \leq K_{2}\left(\delta, \mu_{1}, \widehat{\mu}_{2}\right)\left\|\left(u_{0}, u_{1}\right)\right\|_{\omega_{d}}^{2} .
$$

Summing (7.26) and (7.27), and keeping (7.22) into account, we finally obtain that $\left\|\left(u(t), u^{\prime}(t)\right)\right\|_{\omega_{d}}^{2} \leq L_{1}$ for every $t \in[0, S]$. This contradicts $(7.24)$.

\section{References}

[1] A. Arosio, S. Panizzi; On the well-posedness of the Kirchhoff string. Trans. Amer. Math. Soc. 348 (1996), no. 1, 305-330.

[2] A. Arosio, S. Spagnolo; Global solutions to the Cauchy problem for a nonlinear hyperbolic equation. Nonlinear partial differential equations and their applications. Collège de France seminar, Vol. VI (Paris, 1982/1983), 1-26, Res. Notes in Math., 109, Pitman, Boston, MA, 1984.

[3] S. Bernstein; Sur une classe d'équations fonctionnelles aux dérivées partielles, (Russian, French summary) Bull. Acad. Sci. URSS. Sér. Math. [Izvestia Akad. Nauk SSSR] 4 (1940), 17-26.

[4] G. Chen, D. L. Russell; A mathematical model for linear elastic systems with structural damping. Quart. Appl. Math. 39 (1981/82), no. 4, 433-454.

[5] S. P. Chen, R. Triggiani; Proof of extensions of two conjectures on structural damping for elastic systems. Pacific J. Math. 136 (1989), no. 1, 15-55.

[6] S. P. Chen, R. Triggiani; Characterization of domains of fractional powers of certain operators arising in elastic systems, and applications. J. Differential Equations 88 (1990), no. 2, 279-293.

[7] S. P. Chen, R. Triggiani; Gevrey class semigroups arising from elastic systems with gentle dissipation: the case $0<\alpha<1 / 2$. Proc. Amer. Math. Soc. 110 (1990), no. 2, 401-415. 
[8] F. Colombini, E. De Giorgi, S. Spagnolo; Sur le équations hyperboliques avec des coefficients qui ne dépendent que du temp. (French) Ann. Scuola Norm. Sup. Pisa Cl. Sci. (4) 6 (1979), no. 3, 511-559.

[9] P. D'Ancona, S. Spagnolo; Global solvability for the degenerate Kirchhoff equation with real analytic data. Invent. Math. 108 (1992), no. 2, 247-262.

[10] P. D'Ancona, S. Spagnolo; On an abstract weakly hyperbolic equation modelling the nonlinear vibrating string. Developments in partial differential equations and applications to mathematical physics (Ferrara, 1991), 27-32, Plenum, New York, 1992.

[11] P. D'Ancona, S. Spagnolo; A class of nonlinear hyperbolic problems with global solutions. Arch. Rational Mech. Anal. 124 (1993), no. 3, 201-219.

[12] E. H. DE BRITO; The damped elastic stretched string equation generalized: existence, uniqueness, regularity and stability. Applicable Anal. 13 (1982), no. 3, 219-233.

[13] M. Ghisi, M. Gobbino; Global existence and asymptotic behavior for a mildly degenerate dissipative hyperbolic equation of Kirchhoff type. Asymptot. Anal. 40 (2004), no. 1, $25-36$.

[14] M. Ghisi, M. Gobbino; Derivative loss for Kirchhoff equations with non-Lipschitz nonlinear term. Ann. Scuola Norm. Sup. Pisa Cl. Sci. (5) 8 (2009), no. 4, 613-646.

[15] M. Ghisi, M. GobBino; Spectral gap global solutions for degenerate Kirchhoff equations. Nonlinear Anal. 71 (2009), no. 9, 4115-4124.

[16] M. Ghisi, M. GobBino; Hyperbolic-parabolic singular perturbation for Kirchhoff equations with weak dissipation. Rend. Ist. Mat. Univ. Trieste 42 Suppl. (2010), 67-88.

[17] M. Ghisi, M. Gobbino; Kirchhoff equations in generalized Gevrey spaces: local existence, global existence, uniqueness. Rend. Istit. Mat. Univ. Trieste. 42 Suppl. (2010), 89-110.

[18] M. Ghisi, M. Gobbino; Kirchhoff equations from quasi-analytic to spectral-gap data. Bull. Lond. Math. Soc. 43 (2011), no. 2, 374-385.

[19] M. Ghisi, M. GobBino; Linear hyperbolic equations with time-dependent propagation speed and strong damping. Preprint arXiv:1408.3499 [math.AP].

[20] M. Ghisi, M. Gobbino, H. Haraux; Local and global smoothing effects for some linear hyperbolic equations with a strong dissipation. Trans. Amer. Math. Soc. To appear. Preprint arXiv:1402.6595 [math.AP].

[21] J. M. Greenberg, S. C. Hu; The initial value problem for a stretched string. Quart. Appl. Math. 38 (1980/81), no. 3, 289-311.

[22] A. Haraux, M. Ôtani; Analyticity and regularity for a class of second order evolution equation. Evol. Equat. Contr. Theor. 2 (2013), no. 1, 101-117. 
[23] F. Hirosawa; Global solvability for Kirchhoff equation in special classes of non-analytic functions. J. Differential Equations 230 (2006), no. 1, 49-70.

[24] R. Ikehata, M. Natsume; Energy decay estimates for wave equations with a fractional damping. Differential Integral Equations 25 (2012), no. 9-10, 939-956.

[25] R. Ikehata, G. Todorova, B. Yordanov; Wave equations with strong damping in Hilbert spaces. J. Differential Equations 254 (2013), no. 8, 3352-3368.

[26] G. Kirchnoff; Vorlesungen ober mathematische Physik: Mechanik (section 29.7), Teubner, Leipzig, 1876.

[27] R. MAnfRIn; On the global solvability of Kirchhoff equation for non-analytic initial data. J. Differential Equations 211 (2005), no. 1, 38-60.

[28] M. P. Matos, D. C. Pereira; On a hyperbolic equation with strong damping. Funkcial. Ekvac. 34 (1991), no. 2, 303-311.

[29] T. Matsuyama, M. Ruzhansky; Global well-posedness of Kirchhoff systems. J. Math. Pures Appl. (9) 100 (2013), no. 2, 220-240.

[30] K. Nishinara; On a global solution of some quasilinear hyperbolic equation. Tokyo J. Math. 7 (1984), no. 2, 437-459.

[31] K. Nishinara; Degenerate quasilinear hyperbolic equation with strong damping. Funkcial. Ekvac. 27 (1984), no. 1, 125-145.

[32] K. Nishinara; Decay properties of solutions of some quasilinear hyperbolic equations with strong damping. Nonlinear Anal. 21 (1993), no. 1, 17-21.

[33] K. Nishinara, Y. Yamada; On global solutions of some degenerate quasilinear hyperbolic equations with dissipative terms. Funkcial. Ekvac. 33 (1990), no. 1, 151-159.

[34] K. OnO; On decay properties of solutions for degenerate strongly damped wave equations of Kirchhoff type. J. Math. Anal. Appl. 381 (2011), no. 1, 229-239.

[35] S. I. Pohozaev; The Kirchhoff quasilinear hyperbolic equation, Differentsial'nye Uravneniya 21 (1985), 101-108 (English transl.: Differential Equations 21 (1985), 82-87).

[36] M. Reed, B. Simon; Methods of Modern Mathematical Physics, I: Functional Analysis. Second edition. Academic Press, New York, 1980.

[37] Y. ShibAtA; On the rate of decay of solutions to linear viscoelastic equation. Math. Methods Appl. Sci. 23 (2000), no. 3, 203-226.

[38] Y. YAmadA; On some quasilinear wave equations with dissipative terms. Nagoya Math. J. 87 (1982), 17-39.

[39] T. YAmazAKI; On local solutions of some quasilinear degenerate hyperbolic equations. Funkcial. Ekvac. 31 (1988), no. 3, 439-457.

[40] T. Yamazaki; Global solvability for the Kirchhoff equations in exterior domains of dimension three. J. Differential Equations 210 (2005), no. 2, 290-316. 